JFM

21,1

Received 5 April 2021 Revised 2 August 2021 Accepted 15 September 2021

\section{Studying the relationship between causes and effects of poor communication in construction projects using PLS-SEM approach}

\author{
Yaser Gamil \\ Lulea University of Technology, Lulea, Sweden, and \\ Ismail Abd Rahman \\ University of Tun Hussein Onn Malaysia, Batu Pahat, Malaysia
}

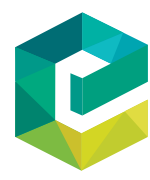

Journal of Facilities Management Vol. 21 No. 1, 2023 pp. $102-148$ pp. 102-148 1472-5967 DOI 10.1108/JFM-04-2021-0039

\begin{abstract}
Purpose - The purpose of this paper is to develop a structural relationship model to study the relationship between causes and effects of poor communication and information exchange in construction projects using Smart-PLS.

Design/methodology/approach - The first method of this research is to identify the causes and effects factors of poor communication in construction projects from the extant of literature. The data used to develop the model was collected using a questionnaire survey, which targeted construction practitioners in the Malaysian construction industry. A five-point Likert type scale was used to rate the significance of the factors. The factors were classified under their relevant construct/group using exploratory factor analysis. A hypothetical model was developed and then transformed into Smart-PLS in which the hypothetical model suggested that each group of the cause factors has a direct impact on the effect groups. The hypothesis was tested using $t$-values and $p$-values. The model was assessed for its inner and outer components and achieved the threshold criterion. Further, the model was verified by engaging 14 construction experts to verify its applicability in the construction project setting.

Findings - The study developed a structural equation model to clarify the relationships between causes and effects of poor communication in construction projects. The model explained the degree of relationships among causes and effects of poor communication in construction projects.

Originality/value - The published academic and non-academic literature introduced many studies on the issue of communication including the definitions, importance, barriers to effective communication and means of poor communication. However, these studies ended up only on the general issue of communication lacking an in-depth investigation of the causes and effects of poor communication in the construction industry. The study implemented advanced structural modeling to study the causes and effects. The questionnaire, the data and concluding results fill the identified research gap of this study. The addressed issue is also of interest because communication is considered one of the main knowledge areas in construction management.
\end{abstract}

Keywords PLS, SEM, Construction projects, Causes, Effects, Poor communication, PLS-SEM

Paper type Research paper

(C) Yaser Gamil and Ismail Abd Rahman. Published by Emerald Publishing Limited. This article is published under the Creative Commons Attribution (CC BY 4.0) licence. Anyone may reproduce, distribute, translate and create derivative works of this article (for both commercial and noncommercial purposes), subject to full attribution to the original publication and authors. The full terms of this licence may be seen at http://creativecommons.org/licences/by/4.0/legalcode

Authors acknowledge the financial support provided by the Ministry of Higher Education in Yemen and the research supports by the university. 


\section{Introduction}

The success of communication in the construction project is essential to produce a successful collaboration and understanding between project parties, which eventually leads to satisfying project completion (Dainty et al., 2007; Hair et al., 2021). It is also required to ensure better quality and performance of the project execution (Günhan et al., 2012). The project management body of knowledge (Guide, 2001) considers communication as a significant component of the project, which plays an essential role in information exchange and mutual collaboration among construction parties.

On the contrary, ineffective, poor and substandard communication among construction parties leads to many undesirable sequences, such as dispute and failure of the project (Hossain, 2009). It is also a significant source of many other negative impacts to project performance and quality (Henderson et al., 2016). The term poor communication is described as the phenomenon of unsuccessful exchange of project information (PI) throughout the lifecycle of the project (Loosemore and Lee, 2002). There are many causes and effects of poor communication, which have been uncovered previously through investigations of literature and exploratory interviews with construction experts. The cause-and-effect factors of poor communication have been classified into their corresponding groups/construct using factor analysis. This article aimed to use the data to develop a structural equation model (SEM) to study the relationships between causes and effects of poor communication in construction projects.

\section{Literature review}

In recent years, the construction industry has expanded significantly and become one of the leading industrial sectors for the development of the society and economy and one of the primary sectors for job generation (Isa et al., 2013). Therefore, its sustainable growth and progress is an indicator of the development of the countries (Dainty et al., 2007). The construction industry has unique characteristics in comparison with other industrial sectors where it is dynamic, multifaceted, complex, multidisciplinary and expandable (Chen, 1998; Dubois and Gadde, 2002; Senaratne and Ruwanpura, 2016). Consequently, these characters are accompanied by many challenges and problems, such as poor quality of construction, poor performance, cost and time overruns, accidents, poor communication and project failure (Abd El-Razek et al., 2008; Alaghbari et al., 2007).

Effective communication in the construction project lifecycle is an indispensable element for the success of the projects because it involves in all the project stages as a method of imparting and exchanging PI (Dainty et al., 2007). For that reason, maintaining a proper process of communication and effective flow of information can be a significant challenge due to the vast amount of information and the number of parties engaging in a single project (Loosemore and Lee, 2002). On that account, poor communication develops and becomes a detrimental phenomenon in construction projects (Zulch, 2012). It is also considered as one of the hindrances to maintaining a steady execution process and effective project delivery (Senaratne and Ruwanpura, 2016).

Poor communication is the term used to describe the unsuccessful delivery of PI, the wrong selection of channeling and improper timing of information distribution (Dainty et al., 2007). In a corresponding finding, a report released by the Project Management Institute (PMI, 2013) revealed that more than half of all project budget risk is due to ineffective communications, improper channeling and inaccurate time management of information dissemination. The report further elaborated, inferior and substandard communications are the primary cause of project failure. Furthermore, the British Standards Institution (BSI, 2003) estimated the cost required to correct defects due to ineffective communication of PI is
Causes and effects of poor communication

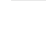


JFM

21,1

104

valued at approximately $220 \mathrm{bn}$ annually in the UK construction industry. It also emphasized that for better communication and sharing information between designers, engineers and contractors would likely to be essential to complete the project on time and budget.

Poor communication in the construction workplace leads to many other negative impacts. Frustration, friction, tense environment and demotivation are among the negative impacts of poor communication (Zulch, 2012). These issues result in poor productivity and efficiency whereby employees tend to overreact upon any matter. Hence, effective communication is necessary to produce a more understandable workplace and facilitate the interaction between employees to ensure successful completion of the project.

Regarding the situation of the Malaysian construction industry, which is the study area of this research, it has a multicultural and multilingual society where different languages are used and different interpretations are developed during the PI exchange (Kuang et al., 2010). Malaysia is one of the most diverse countries due to its multicultural and multilingual societies comprising different ethnic groups and backgrounds whereby the most dominant ethnic groups are Malays, Chinese, Indian and others in which they speak more than one language and bounded to different cultures (Kuang et al., 2010). Malaysian construction sectors involve all the races in the country alongside with international investors and workforce. The workforce is mainly from Bangladesh, Indonesia, Pakistan, Myanmar and other ASEAN countries (Adnan, 2004). Therefore, communication in the industry experiences challenges and barriers to be performed effectively.

Several studies have been introduced on the topic of communication in the Malaysian industry. A study by Valitherm and Rahman (2014) on the communication barrier in Malaysia construction sites found that language barrier is the most severe challenge in construction site whereby supervisors face difficulties to converse and deliver the information and that lead to a safety violation and wrong execution of the project activities. Furthermore, a study by Salleh et al. (2012) on the language problem among foreign workers in the Malaysian construction industry and concluded that the language barrier has led to many accidents. In another perspective by Taleb et al. (2017), investigated the communication management between architects and clients during the design phase and found that it is essential to improve communication management among the architects as a demanding matter to obtain effective performance. Moreover, Pozin et al. (2018) explored the usage of the virtual communication practices in the industrialized building system in the Malaysian construction industry and found that most of the team members are using virtual communication during project implementation due to modern organization reform. Unfortunately, this study is unable to find any research work related to poor communication in the Malaysian construction industry. Hence, more effort and research are required to investigate the issue of communication in the construction industry especially on identifying the causes and effects of poor communication on construction projects success, which is expected to benefit the construction community to understand the issue of poor communication in their respective organizations.

\subsection{Communication in construction projects}

In the construction industry context, communication can be defined as the mutual exchange of PI with the assurance of creating an understandable platform between construction parties (Dainty et al., 2007; Fichet and Giraud, 2007). According to Pérez Gómez-Ferrer (2017), communication is the process of sending and receiving technical and non-technical information through different means and channels and among different managerial and non-managerial levels. PMBOK (Guide, 2001) defined project communication management as "one of the main knowledge areas in project management, which include the processes 
requires to ensure timely and appropriate generation, collection, distribution, storage, Causes and effects retrieval, and ultimate disposition of project information." Communication covers all tasks related to producing, compiling, sending, storing, distributing and managing project records above and beyond it also necessitates an accurate report on the project status, performance, change and earned value (Crowe, 2005). On another side, Emmitt and Gorse (2006) described effective communication as a key management competency to effective leadership and decision-making. Correspondingly, Dingsdag et al. (2006) defined effective communication as the ability to communicate with employees. Emuze and James (2013) described communication as one of the fundamental components of the construction industry, and it is the crucial component to project success.

\subsection{Concept of poor communication in construction projects}

Communication in the construction industry exhibits complexity, heterogeneity and challenges due to the huge amount of PI and the number of parties involved in a single project (Emmitt and Gorse, 2006). Communication in construction projects is arguably one of the most important aspects in project management that supersede others and effective communication is a significant factor for the successful accomplishment of construction projects (Dainty et al., 2007). However, poor communication in construction is a global problem that requires attention to explore solutions and mitigation measures.

The term poor is used by many researchers to describe the phenomenon of ineffective communication in construction projects. Hoezen et al. (2006) used the term to describe the improper and lack of communication among construction stakeholders. BSI (2003) used the term poor as a general concept to describe the inefficient communication and inappropriate exchange of PI in the construction industry. Similarly, Dainty et al. (2007) used the term poor to articulate the concept of improper, inadequate communication and ineffective project communication among construction teams.

Poor communication practices have been recognized as a severe delimiting factor to the success of construction projects (Loosemore and Lee, 2002). Rostami and Oduoza (2017) classified lack of communication as a key risk in the Italian construction industry and Thunberg et al. (2017) categorized the failure of internal communication and external communication as a key problem in the supply chain management in construction projects. From another point of view, poor communication leads to many undesirable effects, such as conflicts, frustrations among construction parties, which subsequently cause tremendous problems, such as unexpected cost loss, delay and litigation between construction teams (Arnorsson, 2012; Tipili and Ojeba, 2014). It also causes errors and reworks in construction projects, which then incur an extra cost for restorations (Arnorsson, 2012; Love and Li, 2000).

On that account, ensuring the right information and instructions are disseminated from all levels of management requires effective communication skills among construction practitioners (Guide, 2001). Hence, keeping adequate communication among all project participants is a key factor in project success (Sinesilassie et al., 2018). For these motives, avoiding the phenomenon of poor communication in the construction industry requires more investigations and research on the cause and consequences (Dainty et al., 2007; Loosemore and Lee, 2002; Rostami and Oduoza, 2017).

In the construction industry, it is a considerable challenge to maintain the effectiveness of communication due to the complex nature of projects and the massive amount of information exerted on a daily basis (Dainty et al., 2007). According to Quill (1995), the most dominant barriers to effective communication are background diversity, different levels of experience and personal barriers. Furthermore, Jureddi and Brahmaiah (2016) classified the 
most common barriers to effective communication, which include, but are not limited to language barriers, psychological barriers, physical barriers and attitudinal barriers. According to Torrington and Hall (1998), the common barriers to effective communication are the individual's frame of reference, which refers to the interpretation of an individual based on his/her reference, stereotyping, which means people may stereotype others according to their own perceptions and cognitive dissonance, which means if someone receives information which is not in line with their beliefs then that will cause a barrier to understand or respond in a positive way which consequently creates communication barriers. Additionally, Ross and Dewdney (1998) identified the barriers to effective communication, which include selective perception: listener only picks the message that supports his or her ideas or opinions, making assumptions: misinterpreting the meaning or feeling rather than listening to the details, giving unsolicited advice: listener gives information before listening to the problem, being judgmental: listener becomes critical of others opinions or point of views in a way that creates a barrier or gap that can not be comprehended from the transmitter's perspectives, acting defensively: the listener defending a position rather than listening to the opinion of another person and failing to understand cultural differences. From another perspective, Buchanan and Huczynski (2019) and Othman and Mydin (2014) outlined the barriers of effective communication as power differences, which happens due to the fact that employees distort communication upward and believe that superiors have a limited understanding of subordinate's needs, gender differences: men tend to talk more, whereas women tend to listen and reflect more, physical surroundings: issues such as room layout, noisy equipment and physical proximity, which affect communication effectiveness, language: variations in language and dialect can affect communication and cultural diversity: different cultures harbor dissimilar expectations as regard formal and informal expectations. Furthermore, Loosemore and Lee (2002) added, the main barriers to effective communication in the construction industry are culture, poor control of diversity, language, poor technical skills and different levels of education among the project team.

\subsection{Importance of communication to project success}

Communication is an essential component for the success of construction projects (Dainty et al., 2007). The success of the construction industry is wholly reliant upon effective communication between individuals, teams and organizations (Pérez Gómez-Ferrer, 2017). Therefore, leveraging efficient communication methodologies has positively impacted project quality, scope clarity and business benefits (Coopers, 2012). According to Zulch (2014), effective communication minimizes the cost, time overruns and improves the quality of work. Furthermore, using proper communication methods and medium help to resolve any design problems and associated conflicts (Tipili and Ojeba, 2014). Also, effective communication smoothens the project implementation from the inception stage to the handover stage and it is essential to understand its important role in the success of the construction industry (Zulch, 2012).

In the context of the organizational project, communication is considered as a core competency, and if it is not executed correctly and connected every member of the project team to a set of consistent strategies and procedures then project outcomes are jeopardized, and the budget incurs unnecessary risk (Dainty et al., 2007). A report developed by Coopers (2012) on the role of communication in project outcomes revealed that effective communication plays an essential role to the success of projects and the report investigated the opinions of executive and project managers in which 9 out of 10 chief executive officers 
agreed that communication is critical to the success of strategic initiatives and considered Causes and effects communication as an integral component of strategic planning and execution process.

Several studies discussed the importance of effective communication in the construction industry. A survey by Fichet and Giraud (2007) on the role of communication toward the improvement of performance found that quality performance depends upon effective communication. Further, the effective change of information and ideas between members of the design team can simplify the design process and provide an efficient outcome (Best and De Valence, 2007). From another viewpoint, Liao et al. (2014) investigated the relationship between communication execution and safety in China and found that communication is a very pivotal factor to manage safety and produces a safe workplace and easy dissemination of safety information.

Effective communication among project team members is vital to a project's accomplishment and conforming quality in construction projects (Lohiya, 2012; Pasley and Kim Roddis, 1994). It plays a significant role in the empowerment of collaboration between project actors (Cheng et al., 2001). To support that, a survey was conducted by Coopers (2012) on the current state of project management disclosed that a $17 \%$ increase in finishing projects within budget was associated due to effective communication. Similarly, communication and coordination are essential elements of organizations (Kornelius and Wamelink, 1998). Unarguably, communication between individuals, organizations and stakeholders is the glue to maintain the project to move forward with minimal hindrances (Emmitt and Gorse, 2006). That being so, more than $70 \%$ of a project manager's time is spent on communication, which is used for communicating with stakeholders and relevant parties in the project (Turley, 2010). On top of that, outlined, communication is the core of management discipline and insisted that more attention must be given to developing communication in the industry (Kent, 1996). Additional significances of effective communication are offered by Armstrong and Taylor (2020) as follows:

- Achieving coordinated results: effective communication assists to smoothly organize the collective actions of team members and the vast amount of information, which further avoids independent actions that lead to incompatibility and mismatched with project objectives and coordinated outcomes.

- Managing change: frequent change is a standard in construction projects, but the presence of effective communication supports to avoid conflict during change and variations.

- Motivating employees: quality of communication from managers can effectively improve the degree of motivation and produce self-initiative individuals among construction teams.

- Understanding the needs of the workforce: effective channeling of communication can facilitate and accelerate the response of the organization to any alert in the workplace.

\subsection{Causes of poor communication in construction projects}

An investigation of several articles on poor communication was carried out to identify the causative factors of poor communication from different literature a total of 35 causes factors were identified and tabulated (Gamil and Rahman, 2017).

One of the common causes of poor communication is the lack of timely communication among project parties. It is one of the leading causes of poor communication in the construction industry, which has significantly resulted in many negative consequences to 
the construction project and further has been confirmed that the less rate of communication among parties during the project can cause time and cost overruns (Badu et al., 2010; Chan and Kumaraswamy, 1997; Hoezen et al., 2006; Sambasivan and Soon, 2007). Additionally, lack of communication can cause rework and a range of other construction performance deficiency (PER) (Emuze and James, 2013). A comparative study on causes and effects of delay in Nigerian and Iranian construction projects by Oshodi Olalekan and Rimaka (2013) ranked lack of communication between construction parties from the contractor's perspective based on its importance as the 11th and 12th factors of delay for Nigeria and Iran, respectively, however, the ranks from the consultant perspective were 20th and 13th for Nigeria and Iran, respectively. From another viewpoint, a study conducted by Darvik and Larsson (2010) on the impact of material delivery deviations on costs and performance in construction projects showed that quality defects and delivery deviation of materials occurred due to lack of communication among relevant parties and communication failure. Therefore, it was suggested, stakeholders must put more concern in the enhancement of communication regularity, means and methods. PMI (2013) investigated the importance of frequent communication on the overall performance of organizations. It was found that high performing organizations are better at communicating key project areas, including objectives, budget, schedules, scope, outcomes and business benefits. It was also added, frequent and sustained communication is essential to maintain the project activities flow over the stipulated schedule assigned for the whole project; therefore, a delay is diminished and quality is improved.

Another reason for poor communication is the lack of a communication system, which is defined as the complete structured platform of the communication process in the whole organization. If the communication system is established, then the information dissemination will be easily managed (Ballan, 2011). A communication system is also called an information management system and is used to manage information flow and dissemination time in the construction industry and a complete reporting system to administer the communication and feedbacks that can keep the process in balance by reacting to any deviation during construction projects (Affare, 2012). In a qualitative study by Nguyen and Chileshe (2013), it was found that lack of effective communication system in the implementation of projects ranks number 12 as 1 of the critical factors ranking causing failure of construction projects in Vietnam. Therefore, the lack of an effective communication system hampers the process of communication in the construction industry (Olanrewaju et al., 2017). For that reason, a complete system needs to be implemented to manage information of the project from the planning stage to the completion stage of construction projects (Dainty et al., 2007).

Communication skills are essential for effective communication and lack of communication skills is a reason for poor communication, especially in construction projects where skills required, are technical, interpersonal and social skills. Everyone in the construction project is expected to have these skills to provide quality work and one of the interpersonal skills is effective communication (Dainty et al., 2007). Interpersonal skills include communication skills play an indispensable role in the success of the project (Günhan et al., 2012). Typically, parties involved in construction possess different communication skills, which also depend on their qualification and cultural background. These differences cause concurrent misunderstandings in the construction and delivery stages of the project (Cheng et al., 2001).

Language barrier is also one of the main causes of poor communication in construction projects because the construction industry with a unique characteristic is diversified and comprising many people of different backgrounds, cultures and languages (Abd El-Razek et al., 2008; Zhang, 2010). Construction parties speak different languages and have different cultural backgrounds; these differences if not well-managed can cause communication 
failure in a construction site (Dainty et al., 2007). Most of the time, misunderstanding among construction practitioners may occur and that could lead to poor workmanship and productivity (Othman and Mydin, 2014). PMI (2013) reported that the organizations have difficulties in communicating with appropriate levels of clarity and details are likely to be caused by the difference in languages to understand project-specific technical terminologies. The data confirms that an average of four out of five projects that are communicated with sufficient clarity and details expressed in the language of the audience meet their original goals and intent in comparison to just over half of projects when communication is not sufficiently clear and detailed. An investigation conducted by Tipili and Ojeba (2014) on the effects of communication in construction project delivery in Nigeria, which focused on 40 professionals working in the construction industry in Bauchi, Nigeria and were asked to evaluate based on the low, medium and large scale on how language can hinder communication on construction. The study has shown that $33 \%$ answered medium, which means that the language used by operatives is essential for effective communication on site.

Not forgetting that, poor communication channeling is one of the causes of poor information dissemination where communication is managed by channels in all the organizations, hence each department or section in the organization is responsible for disseminating the related information to formulate a complete communication system. However, due to the complexity in the construction industry, several concurrent communication problems occur because no proper channeling is adopted to manage the communication process (Fichet and Giraud, 2007). Communication in construction is seriously obstructed due to a lack of appropriate data channels, inappropriate channels and inaccurate data transfers (Lee and Bernold, 2008). Also, unclear channels of communication cause project delays (Tipili and Ojeba, 2014). It is, therefore, essential to standardize the channels of communication in the construction industry to expedite and ease the communication process. In reality, the selection of the correct channel in communicating PI is delicate and intricate due to the fragmentation and complexity features of the construction industry. Fichet and Giraud (2007) explained the criteria for selecting an appropriate medium and channel for successful communication, which includes the possibility to get feedback immediately, the potential offers by the mean of communication to express different elements of communication, such as body language, facial expressions and tone of voice, and the option offers by the medium to focus on a particular person. Furthermore, the selection of inappropriate medium or channels can also create communication breakdown. It can occur when a receiver is expected to select the wrong medium and happens on a construction site, which leads to poor communication (Khahro and Ali, 2014). Therefore, to avoid miscommunication the selection of an appropriate channel is essential for the success of communication.

In different prospective possessing different levels of education and experience can cause difficulty in communication, it can create a misunderstanding environment whereby the receiver or sender lacks the appropriate methods of communication. Communication is basically a vertical process in which top management has to communicate with lower therefore if they possess different levels of education then poor communication and misinterpretation are resulted (Dainty et al., 2007). Further, lack of communication procedures and training refers to the shortage of sponsored training for employees on the communication process and method. Hence, it creates difficulty in conducting information exchange and communication (Khahro and Ali, 2014).

In the aspect of lack of support for advanced communication technology, companies tend to downgrade the impact of improper communication and do not allocate a budget to support the use of advanced technology in facilitating communication, which may contribute to the deficiency of the communication process (Ahuja, 2007). However, then
Causes and effects of poor communication

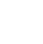


again malfunction of technology is an issue in the construction site, it is basically common to face during the project execution whereby the location of construction sites maybe faces short in supply of electricity and network coverage and these cause malfunctioning communication technology (Tai et al., 2009).

Concerning the diversity of culture, construction companies involve many people from different cultures and backgrounds, which creates an improper interpretation of communication. Hence, it creates a tense environment and requires more concern to resolve this issue (Loosemore and Lee, 2002). It is also the same with diversity in ethnical groups in the project whereby the receiver or the sender limits the frame of communication to his/her ethnics, which outweighs the concern of creating successful communication (Günhan et al., 2012). Additionally, individual barriers can also create miscommunication, which is meant the communicator is creating his own barriers and lack of confidence can also be a personal barrier of being shy or undetermined cause poor communication (Dainty et al., 2007). Gender difference can be a hindrance to effective communication, it refers in this context to the cultural barriers in which men prefer to seek information only from men and the same from women and that creates miscommunication (Waziri and Khalfan, 2014).

Essentially, lack of communication plan is one of the causes of poor communication, which refers to the plan of communication which has to explain the details of information flow in terms of the time of communication, type of information and person in charge of disseminating the information and without prior communication plan, communication on construction site is improperly managed and administered therefore it causes poor communication (Lee and Bernold, 2008). Improper communication time management is the main cause of poor communication, which is referred to as the improper plan of time which causes pressure when it comes to the construction site on a sudden basis. It is also referred to as the equivalence of selecting the right information at the right time (Dainty et al., 2007). Poor planning and coordination point to the overall plan and coordination of the project in terms of scheduling and monitoring of construction activities. Hence, improper planning results in poor communication (Bandulahewa, 2015), poor communication management refers to the deficiencies of managing the huge amount of information in terms of archiving, storage and dissemination. It also causes hassle to answer what type of information is needed when it is needed and how it is transferred (Dainty et al., 2007).

Inaccessibility of PI also causes miscommunication and refers to the restrictions between construction teams involved in the construction can hinder the communication process, which also creates a shortage of information especially in emergency communication, which then leads to augment the miscommunication among the parties (Thorpe and Mead, 2001).

Frequent change of project contract can also cause poor communication, which is common to the construction industry in which the terms change due to the nature of the project or the requirement of the client and need of modification due to encounters during the execution of the project on the construction site. These changes of contract or design or methods can cause poor communication (Bandulahewa, 2015; Enshassi et al., 2006). Lack of clear communication objective denotes that the person in charge of communication is not aware of the targeted goals of communication, which then causes wrong selection of information and creates misunderstanding (Olanrewaju et al., 2017). Also, without a clear purpose of communication, it leads to uncertainty of the message and confusion between the transmitter and receiver (Baguley, 1994), Apart that, lack of mutual trust among construction parties is implied by less transparency and credibility, which may be caused by cultural and ethical diversity. Trust among parties is a key to successful communication and understanding (Dainty et al., 2007). 
From another point of view, weak organizational structure refers to the limited authority of personal in terms of communication, which resembles the characteristics of functional organization structure and improper definition of activities and task allocation can cause poor communication. Hence, a good organizational structure provides a firm foundation for the standards procedures and communication process (Tai et al., 2009).

Contractual barriers refer to the restrictions of obtaining the PI due to the terms in the contract in which the contract may assign certain tasks to a certain party, thus it restricts others from interferences and results in miscommunication occurrence (Dainty et al., 2007). Also, lack of representation for project stakeholders refers to the absenteeism of construction meetings and that is interpreted by lack of being accountable, which can cause poor communication (Thomas et al., 1998).

From the technical prospective, poorly detailed drawing refers to the lack of clarity and specifics that explain the technical drawing especially to personnel with less experience in translating the drawing into practice (Aulich, 2013). Also, incorrect instructions or technical information can generate miscommunication and that results to cause reworks due to the wrong execution of certain project activities (Aulich, 2013).

\subsection{Effects of poor communication to construction projects}

Poor communication has many negative impacts on the project. It is one of the main factors triggering the failure of construction projects (Tipili and Ojeba, 2014). As a result of poor communication, many undesirable effects and consequences caused, which mainly includes, but not limited to cost overruns, time overruns, dispute and others. Furthermore, ineffective communication leads to unproductive outcomes (Gamil et al., 2019). According to PMI (2013) report on the investigation of communication in organizational perspectives, which shows the differences between highly effective communicators and minimally effective communicators in meeting original goals, on-time delivery and within budget. It is shown that highly effective communication results in $80 \%$ of meeting initial goals, $71 \%$ achieving on-time project delivery and $76 \%$ within budget planned. However, minimally effective communication results in declination of meeting goals, time and cost outlined. Figure 2.6 illustrates the relationship between the frequency of effective communication and project success components. It is shown that highly effective communicators achieve better results in meeting original goals, on-time delivery and within the stipulated budget.

Time overrun can be a consequent of poor communication, which is known as a delay and is referred to as time beyond the completion date assigned in the contract during the planning stage of the project (Chan and Kumaraswamy, 1997; Dainty et al., 2007). It is classified as the most frequent problem in the construction industry worldwide (Alaghbari et al., 2007). It commonly causes adverse effects on project success (Faridi and El-Sayegh, 2006). Literature has revealed that poor communication is one of the main factors contributing to delay in construction (Afshari et al., 2010; Ren et al., 2008). It is certain that, without proper communication on the objective, it can cause a delay (Olawale and Sun, 2010). Delays triggered by poor communication can be in the form of slow information flow, improper communication channels, wrong design, wrong interpretation, reworks and others (Batool and Abbas, 2017; Dainty et al., 2007; Love and Li, 2000; Tipili and Ojeba, 2014). Poor communication in the construction industry can cause time overrun in many forms and aspects, some of these include the following:

- Late and slow dissemination of information: if communication is not active, then the flow of information is slow, which contributes to cause time overrun. Slow response to inquiries also causes a delay in progress (Guide, 2001).
Causes and effects of poor communication 
JFM

21,1

- Lack of communication during the early stage of the contract causes continuous changes of the contract (Shehu et al., 2014).

- Inadequate communication among construction teams during execution leads to cumulative communication and causes poor management of information, which consequently causes a delay in the work process (Shehu et al., 2014).

- Communication is not correctly carried out due to the weak organizational structure of construction (Dainty et al., 2007).

- Ineffective or unprepared reporting systems can cause poor communication, which leads to more time in managing the PI (Tipili and Ojeba, 2014).

- Improper channels of communication, especially during emergency calls or during reporting of any work process can cause a delay in work progress (Tipili and Ojeba, 2014). Faulty transmission of the message via an inappropriate medium or channel. It is happening when the receiver engages in too much information from different channels and mediums that consequently causes poor communication (Dainty et al., 2007).

- Technology malfunction causes poor communication, which subsequently triggers the delay of the project (Dainty et al., 2007).

Following that, a dispute, which is a phenomenon of disagreement on a specific set of terms and in the construction industry is commonly known as an endemic and chronic issue (Sinha and Wayal, 2007). It occurs when project parties disagree or misinterpret the contractual terms and, thus results in continuous dispute and that significantly have an impact on the main project components, such as cost, time and quality of construction projects and in some cases results in the failure of the project as a whole (Gebken and Gibson, 2006). Poor communication among the construction parties is one of the main causative factors of dispute. It is, therefore, the main consequence of ineffective communication (Adnan, 2004; Adnan et al., 2012). Effective communication is vital to improve the relationship between the project team (Adnan et al., 2012). Khahro and Ali (2014) studied the indirect causes of dispute in the Pakistan construction industry and found that poor feedback system and negligence are ranked as the first and second indirect causes of the conflicts with an average mean value of 2.7 and 2.65, respectively, whereas the lack of communication procedures and non-adherence of communication procedure set are classified as number 5 and 6 with an average value of 2.38 and 2.34, respectively, as the trivial or indirect cause of the dispute in the construction projects.

Effective communication is the most powerful motive to strengthen the relationship and understanding among the parties of the project (Adnan et al., 2012). On the contrary, ineffective communication is one of the main causative factors of dispute existence in the construction industry (Sinha and Wayal, 2007). According to Malleson (2013), the potential source of conflict varies depending on the project type and most likely arises from the lack of communication and the absence of transparency among construction parties. A study by Cakmak and Cakmak (2013) on the identification of causes of disputes in the construction industry considered lack of communication among construction parties as the leading cause of dispute occurrence.

Consequently, collaboration among construction parties is essential to produce successful projects through the concept of integrated project delivery (Kent and BecerikGerber, 2010). Nevertheless, it has been a hindrance due to the existence of a dispute and it becomes challenging to maintain a good relationship and cooperation among project parties (Kassab et al., 2006). 
In regard to cost overrun, it refers to the project failed financially to achieve its objective Causes and effects (Abdullah et al., 2009; Rahman et al., 2013). Cost overrun occurs when a project's cost exceeds the estimated cost and causing major conflict and litigation, which remarkably lead to project failure (Shehu et al., 2014). There are several causing factors of cost overrun issue in the construction industry. Poor communication among construction parties is one of the leading causes of cost overrun in the construction industry (Rahman et al., 2013). A study by Alhomidan (2013) in Saudi Arabia had identified 41 cost overrun factors in several road construction projects and found that most of the critical factors causing cost overruns are internal administrative difficulties, poor communication among construction parties, payments deferment and delays in decision-making. In another investigation by Bassioni et al. (2013) uncovered that lack of communication and coordination between design participants of different backgrounds documented a higher relative impact to cost overrun in the design phase of the Egyptian construction industry. It was also indicated that communication difficulties during the execution of the project could directly lead to an increase in unnecessary expenditures (Sinesilassie et al., 2018). As in time overrun factors, again poor communication is regarded as an essential factor, which leads to cost overrun. However, not many research studies on factors causing cost overrun as compared to time overrun.

Based on the findings, cost overrun can be caused by poor communications in the following forms:

- Rework: in construction work, some changes of design are reported after the execution of specific jobs due to poor management of communication, the instructions are delivered late then demolishing has to take place to restructure the work as instructed, which requires new materials and labor (Love and Li, 2000).

- Poor details of construction drawing: basically, detailing is meant by the process in which the designer conveyed to a contractor by the mean of technical documentation. Poor detailing causes cost overrun if the drawing is misinterpreted or transferred wrongly due to improper communication among designers and engineers on-site (Dainty et al., 2007).

- Lack of communication among construction parties: it can cause adverse outcomes in terms of cost by creating a delay, which subsequently causes cost overrun due to incomplete tasks on schedule (Dainty et al., 2007).

Regarding rework, which is a common issue in the construction industry (Nagapan, 2014). It is defined as the unnecessary work or activities of redoing or replenishing a job that was essentially executed incorrectly for the first time (Love and Li, 2000). Several descriptions of rework in construction can vary depending on the type of project. Fayek et al. (2003) classified the form of rework as quality deviations, non-conformance, defects and quality failures. Rework was described as the process of doing activities more than once in the field with no prior change of scope, which has been set by relevant parties (Fayek et al., 2003). As a result, rework is a source of other problems, such as waste generation and cost overruns (Nagapan, 2014).

Rework in general, requires an additional budget to repeat the work to satisfy the quality and set of standards. According to research conducted by Nylén (1996) to investigate the significant causes of rework in the construction of highway projects and found that a total of 23 failure factors during the production phases were identified, which accounts for $10 \%$ of the whole production cost and $51 \%$ of these failures were originated from communication problems between consultant and clients. Another study on quality failure in the 
construction industry was introduced by Hammarlund and Josephson (1991) specified that $13 \%$ of quality failures are induced by poor communication among construction parties. It was also identified that in the UK, more than $30 \%$ of construction cost overrun is caused by rework occurrence (Ofori and Ekanayake, 2000). Furthermore, in Australia, it was reported to be up to $35 \%$ of the total cost of the project and contributed as $50 \%$ of the total cost overruns in construction projects (Aziz and Hafez, 2013). Therefore, it was suggested, proper communication platforms must be implied and proper identification of client requirements to minimize rework occurrence (Nagapan, 2014). A case study by Abdul-Rahman (1995) investigated the cost of non-conformance during a highway project and found that poor communication is classified as one of the main factors to non-conformance in which it causes poor quality in construction projects requires the extra cost to rectify non-conformance.

Another study conducted on causes of defects in the South African housing construction industry: perceptions of built-environment stakeholder, which introduced 17 causes of errors in the construction industry and lack of communication between designer and contractors was ranked 11th in respect to its importance caused to the occurrence of defects and required a rework process to rehabilitate the defects (Buys and Le Roux, 2013).

Subsequently, accident on the construction project site. It remains a critical challenge to the industry due to the nature of work in a construction site, the improper practice by labors, safety standards violation and incorrect delivery of PI (Chong and Low, 2014; Dingsdag et al., 2006). Inappropriate communication and wrong delivery of technical information cause accidents in the project site (Chan and Kumaraswamy, 1997). According to Haslam et al. (2005), Salleh et al. (2012), most of the accidents are caused by ineffective communication between the workforce and technical expertise accompanied by poor supervision and inconsiderate adherence to safety regulations.

Referring to the effect of poor communication in creating demotivated workforce, it has been discussed by many researchers that an agitated workforce and negative impact on creativity are the result of poor communication and have negatively impacted the overall performance of the project. The way instructions are being disseminated can affect the workforce negatively if not properly communicated with effective skills. Hence, it requires more attention to improve communication in terms of process and mediums (Dainty et al., 2007; Emuze and James, 2013).

Poor teamwork is also a negative impact of poor communication whereby the individual tends to rely on others to do his own job rather than prominently participating to accomplish the task (Aiyewalehinmi, 2013; Salleh et al., 2012).

Poor communication also leads to a late response to the disaster, whereby during emergency time more information is required to respond to the situation. If there is no clear system of communication, in the time of disaster or accidents time is wasted to decide on the right party before actions are taken (Apolot, 2011; Liao et al., 2014).

Poor productivity is also an effect of poor communication between the project team in the form of deficiency in work in terms of changes of contract or drawing details. The workforce also faces situations where the task is incomplete and of poor quality, which, consequently, resulted in cost and time overrun (Ali and Wen, 2011).

Misunderstanding refers to a factual situation whereby the receiver misreads the information, which leads to mislead due to poor communication. Misunderstanding also leads to misinterpretation and misuse of the information, which leads to an agitated communication environment (Cheng et al., 2001; Emuze and James, 2013).

Design errors are also caused by poor communication whereby the designer receives wrong information and leads to design wrongly, which then creates an undesirable situation 
that requires rehabilitation and correction (Oluwaseun Sunday and Olumide Afolarin, 2013; Causes and effects Sambasivan and Soon, 2007).

Low satisfaction among construction party is also caused by poor communication. If the project parties are not happy with the outcome then affects the collaboration and cooperation to accomplish the project (Hoezen et al., 2006).

Frequent remedies in design and plan are also the sequence of poor communication between project parties. The remedies required to achieve conformance on the requirement are incurred with extra cost and that leads to other negative sequences (Dainty et al., 2007).

Similarly, waste generation is also caused by improper communication and information exchange and that also incurred extra cost and time to perform rehabilitation (Alwi et al., 2002). Poor communication also results in the unclear selection of channels, poor risk management, poor project documentation, poor planning and affects the design and execution process. These causes and effects of poor communication need to be correlated to introduce a clear understanding of the relationship and a global view of the issue of poor communication as well.

\section{Contribution of the study to body of knowledge}

The published academic and non-academic literature introduced many studies on the issue of communication includes the definitions, importance, barriers to effective communication and means of poor communication. However, these studies ended up only on the general issue of communication lacking an in-depth investigation of the causes and effects of poor communication in the construction industry. Hence, this study developed an SEM model to explain and uncover the relationships among causes and consequences of poor communication in construction projects, which have been identified from previous literature (Gamil and Rahman, 2017). The model helps to better understand the phenomenon of poor communication and its impact on project failure.

Furthermore, the model helps to visualize and comprehend the issue addressed in a complete approach. The study filled the knowledge gap related to cause-and-effect factors of poor communication in the construction industry by establishing the relationships between the factors using advanced multivariate analysis. The model is expected to be used to quantify the impact of poor communication on the construction project failure and evaluating the health status of communication and information exchange in the project's settings.

\section{Concept of partial least square-structural equation model}

SEM is developed using the approach of partial least square (PLS) (Bowen and Guo, 2011). These combinations of methods result in producing a basic technique called PLS-SEM (Rigdon et al., 2010; Wong, 2013). It is a common approach used to analyze multi-variables (Garson, 2012; Hair et al., 2021). There are two common approaches in SEM known as CBSEM and PLS-SEM (Hair et al., 2021). Covariance Based structural equation modeling (CB-SEM) is the covariance-based approach and is used to confirm or reject theories. However, Partial Least square structural equation modeling (PLS-SEM) is used to develop theory in exploratory research (Astrachan et al., 2014; Garson, 2012; Hair et al., 2021). In this research, poor communication cause and effect factors are a form of an exploratory study, therefore, PLS-SEM is suitable to be adopted in this research. Generally, SEM comprises two models, which are an outer model and an inner model. The outer model is also called a measurement model or manifests or items and inner models are also called a structural model (Bowen and Guo, 2011; Hair et al., 2021). The outer model shows the relationships between the items and the constructs, whereas the inner model depicts the relationship 
between independent variables (IV) and dependent variables (DV) (Hair et al., 2021; Rigdon et al., 2010).

\section{Adequacy of sample size}

To develop SEM, it is imperative to confirm the adequacy of the sample size used to develop the model (Iacobucci, 2010). According to Hair et al. (2021), the sample size is an essential element in improving the reliability of the model. There have been many types of research on defining the number of sample sizes. According to Kock and Hadaya (2018), the adopted rule of thumb is the 10-time rule as a method to simplify the process of size estimations. However, it is considered an imprecise estimation (Hair et al., 2021). Furthermore, researchers over the past decade used different sample sizes, which depends on the number of variables, the possibility of attaining data and the nature of the research. In construction industry research, PLS-SEM has been extensively used by many researchers. Memon and Rahman (2014) used PLS-SEM to model the causes of cost overrun in the construction industry and the total sample used was 231. Nagapan (2014) developed SEM to study the causes and effects of construction waste generation in the construction industry and collected 277 responses. About the adequacy of sample size in this research, there is no specific size of the sample, however, it was suggested by Hair et al. (2021), the sample size is estimated to be 10 times the number of structural paths to the DV, which are 10 in this study and the total minimum size is 100 accordingly. As the valid sample size collected for this study is 262 which is more than what is suggested by Hair et al. (2021), therefore, the sample size is adequate to develop SEM and the more sample size improves the goodness-of-fit for the model (Coughlan, 2008; Iacobucci, 2010).

\section{Model development and assessment process}

This section presents the development and assessment procedures adopted in generating and assessing the structural model.

Figure 1 shows the overall process involving the development and assessment of SEM. It consists of five major steps, which include hypotheses development and assigning path model, data inputs, running algorithms, assessment of measurement and structural model and testing hypotheses and validation of the results.

\subsection{Hypothetical model}

The first step toward developing SEM is to create a hypothetical model by assuming the path model to predict the relationship between constructs. To initiate that, the previous findings serve as a baseline of the start. According to a study by Nagapan (2014) on the causes and effects of construction waste, the model was an integral between causes and effects. Furthermore, Abusafiya and Suliman (2017), developed PLS-SEM to study the relationships among causes and effects of cost overrun in a construction project in Bahrain and considered the cost overrun as a dependent variable, however, the causes and effects are IV. In this study, the hypothetical model is developed based on the group established by factor analysis. Figure 2 presents the proposed path model and its hypotheses.

According to Figure 2, a total of 12 hypotheses are proposed for the model. These hypotheses represent the independent latent variables which are the causes, however, the effects depict the dependent variable. To accept or reject these hypotheses, the structural model must go through the assessment process in the following subsections. The next step is to transform the hypothetical model into Smart-PLS to represent the relationships among the variables. 


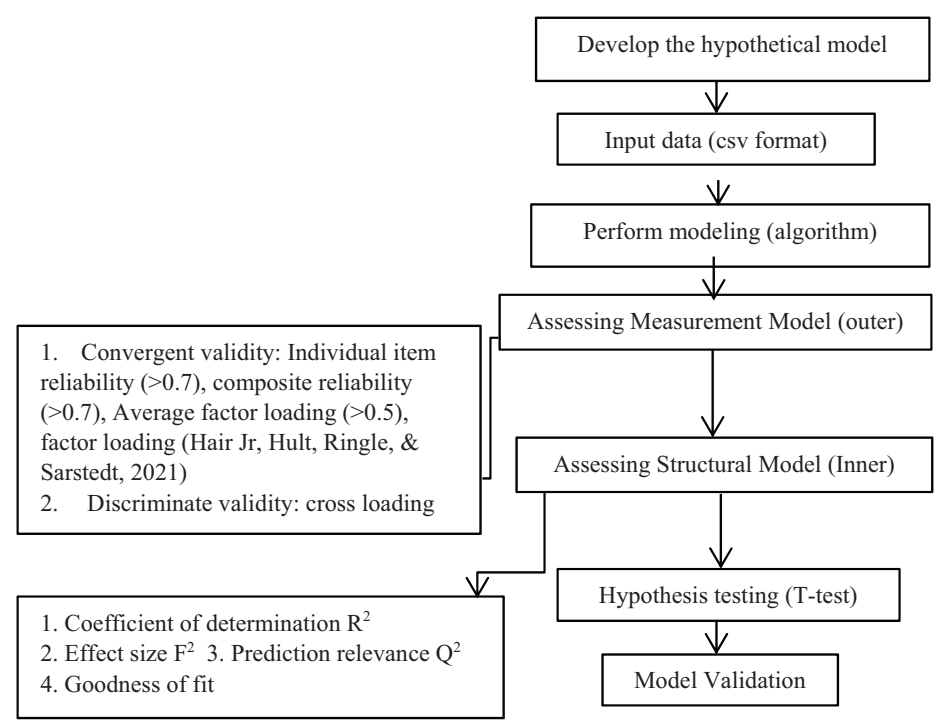

Causes and effects of poor communication

1. Convergent validity: Individual item reliability $(>0.7)$, composite reliability $(>0.7)$, Average factor loading $(>0.5)$, factor loading (Hair Jr, Hult, Ringle, \& Sarstedt, 2021)

2. Discriminate validity: cross loading

2. Effect size $\mathrm{F}^{2}$ 3. Prediction relevance $\mathrm{Q}^{2}$

4. Goodness of fit

Model Validation

Figure 1.

Processes of model development and assessments

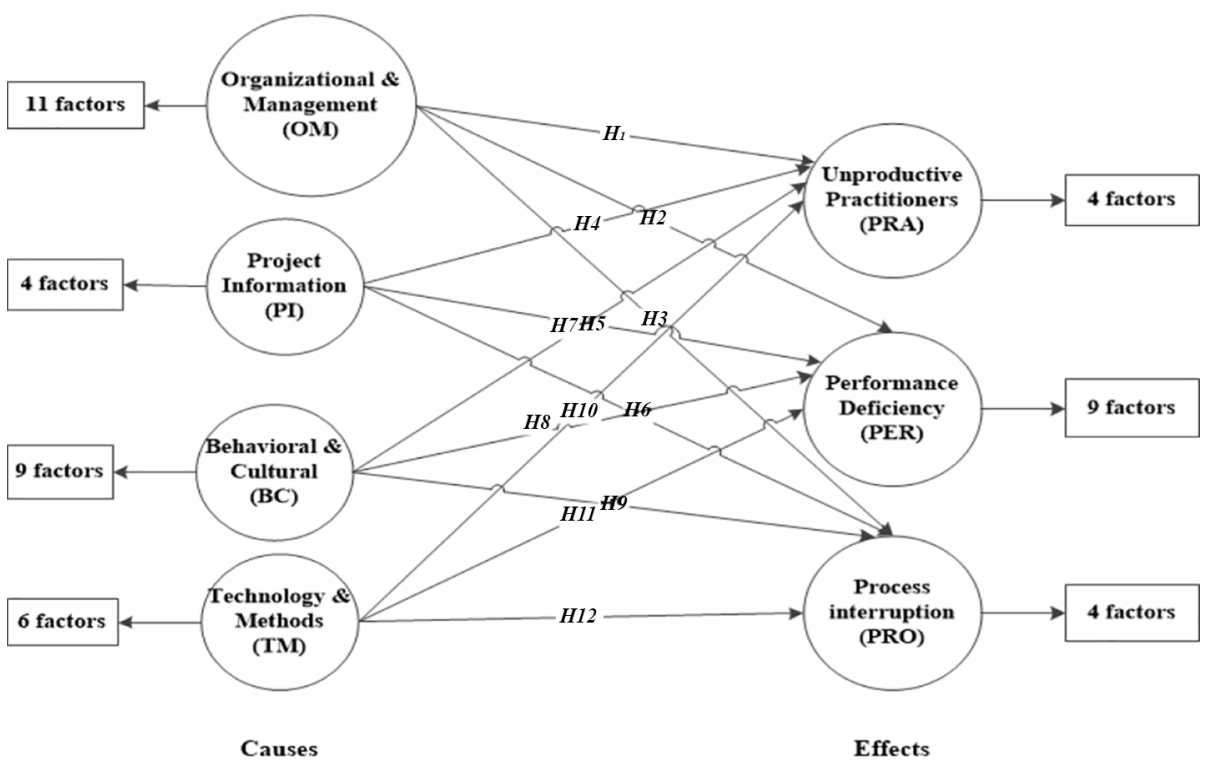

Figure 2. Hypothetical model of poor communication causes and effects relationships

\subsection{Sampling for the survey}

The targeted respondents for this study are construction practitioners from all the management levels and different project parties, which include contractors, clients and consultants in Malaysia. Construction practitioners in this study are referred to as company directors, project managers, architects, project engineers, site engineers and quantity surveyors (Enshassi and Al Swaity, 2015). 
Random sampling technique is widely used in construction research where the sample is randomly selected from the population based on non-zero probability. This technique is considered effective because it produces a sampling representative of the population by avoiding any voluntary response bias (Sandelowski, 2000). All population has the probability of equal chance of being selected as the sample and provide accurate representation for the broader population (Sharma, 2017). Therefore, this technique is adopted to select the participants for this study. The method to determine the sample size of an unlimited population is adopted from Al-Najjar (2008) and Enshassi and Al Swaity (2015) to calculate the sample size:

$$
\mathrm{SS}=\frac{\mathrm{Z}^{2} \times \mathrm{P}(1-\mathrm{P})}{\mathrm{C}^{2}}
$$

Where:

$\mathrm{SS}=$ sample size;

$Z=Z$ value (1.96 for $95 \%$ confidence level);

$\mathrm{P}=$ percentage picking a choice, expressed as a decimal ( 0.5 used for sample size needed);

$\mathrm{C}=$ margin of error $(9 \%)$, maximum error of estimation, which can be $9 \%$ or $8 \%$ (Memon and Rahman, 2014); and

$\mathrm{SS}=\frac{1.96^{2} \times 0.5 \quad(1-0.5)}{0.09^{2}}=118.57 \cong \approx 119$ (As the minimum SS).

To check the marginal error value, the following formula is being used (Enshassi and Al Swaity, 2015). The maximum margin of error for a 95\% confidence level $\approx \frac{1.96}{\sqrt{\text { SS }}}=\frac{1.96}{\sqrt{119}}=$ $\mathbf{0 . 1 8}>\mathbf{0 . 0 9}$. Hence, the margin is acceptable. In this study, the final sample size obtained is 262 respondents.

\subsection{Data input}

The study finalized 262 respondents as core data for generating the model. The respondents are construction practitioners who are working in the Malaysian construction sector. A five-point Likert type scale was used to assess the significance and severity of cause-and-effect factors of poor communication. The final numbers of cause-and-effect factors are 30 and 17, respectively, which makes a total of 47 manifests and this yields $262 \times 47=12,314$ data used to develop the SEM. These data were keyed into SPSS and then saved in a new file as CSV to be compatible with Smart-PLS. The data is then imported into Smart-PLS for further analysis.

\subsection{Path model creation in Smart-PLS}

After drafting the hypothetical model, the items/manifests are then assigned to the relevant independent variable by importing the related data. Figure 3 shows the screenshot of the model after assigning the manifest.

Figure 3 illustrates the model after assigning the manifests to their corresponding latent variables. The color of the LVs turned to blue after assigning the manifests and this shows all the LVs are active for further analysis. The total number of manifests is 47 items.

\subsection{Model execution process}

After assigning all the manifests to their associated latent variable, the following step is to perform the modeling process. The primary step is to run the PLS algorithm to calculate the loadings in each manifest. The main parameters calculated from the algorithm are the assessment criteria, which include both the outer model (measurement model) and the inner model (structural model) (Memon and Rahman, 2014; Nagapan, 2014). 


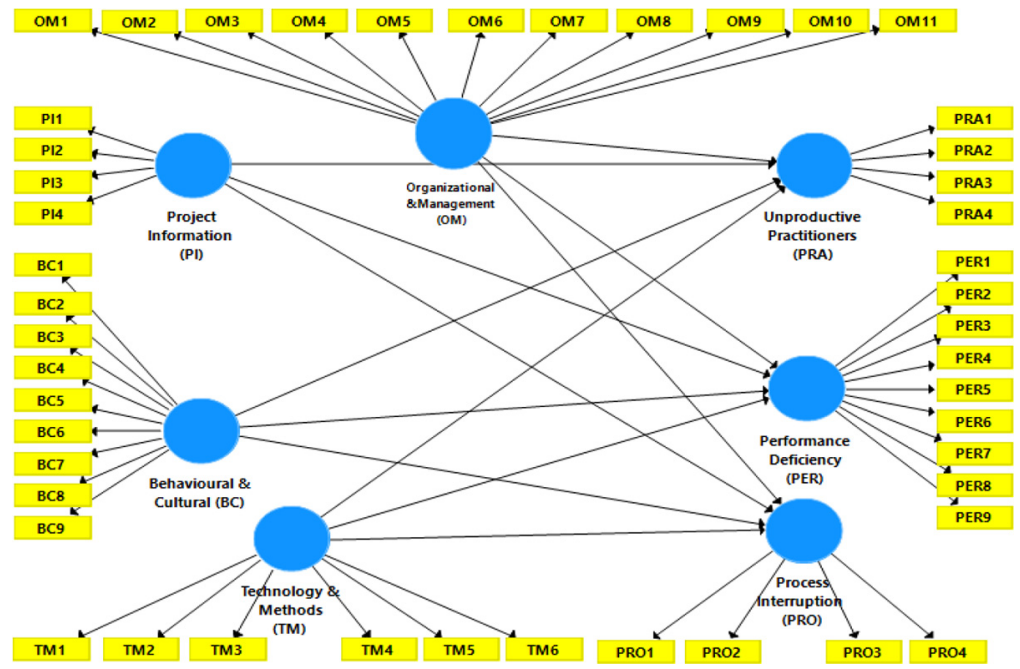

Causes and effects of poor communication

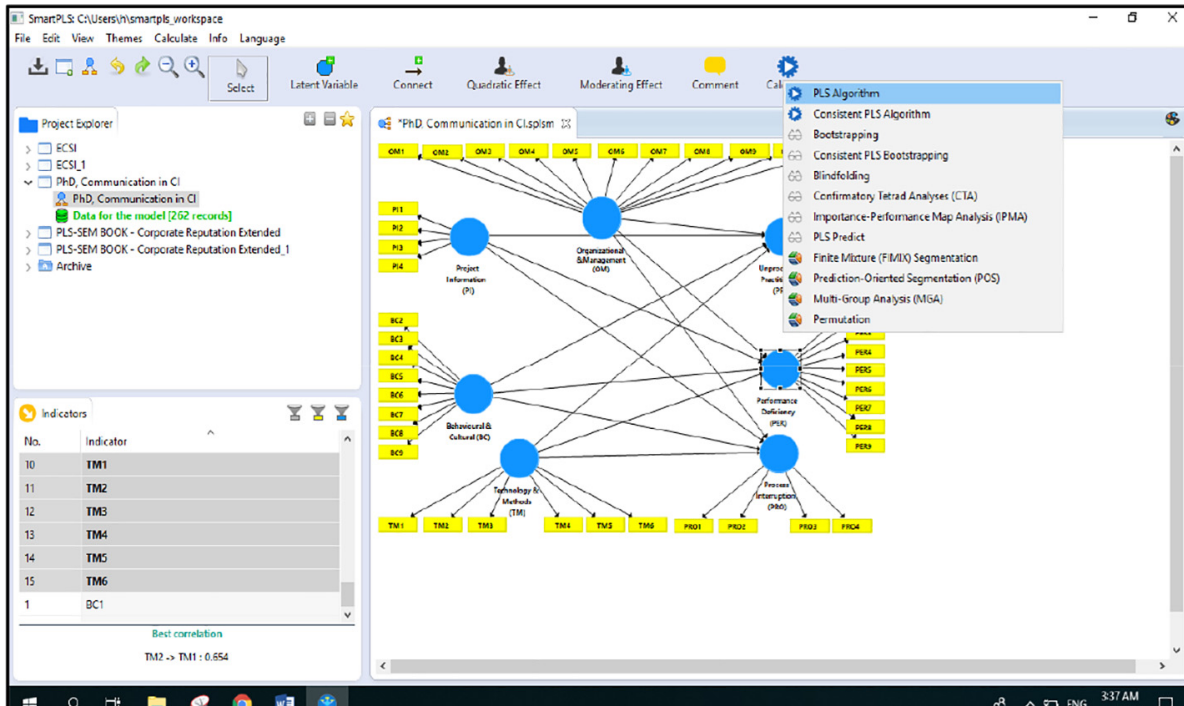

Figure 4.

PLS algorithm simulation (Smart-PLS output)

Figure 4 shows the screenshot extracted from Smart-PLS software to exhibit the process of running the PLS algorithm. The following step is to perform the assessment process of the model regarding its reliability and adequacy of the relationship among the manifests. The next section explains the criteria of model assessments.

\subsection{Criterions of model assessment}

To assess the model's reliability and validity, there are several guidelines and standards developed by the previous researcher for both the model's inner and outer models. Table 1 summarizes the standard model assessment guidelines. 
JFM
21,1

120

Table 1.

Model assessment criterions
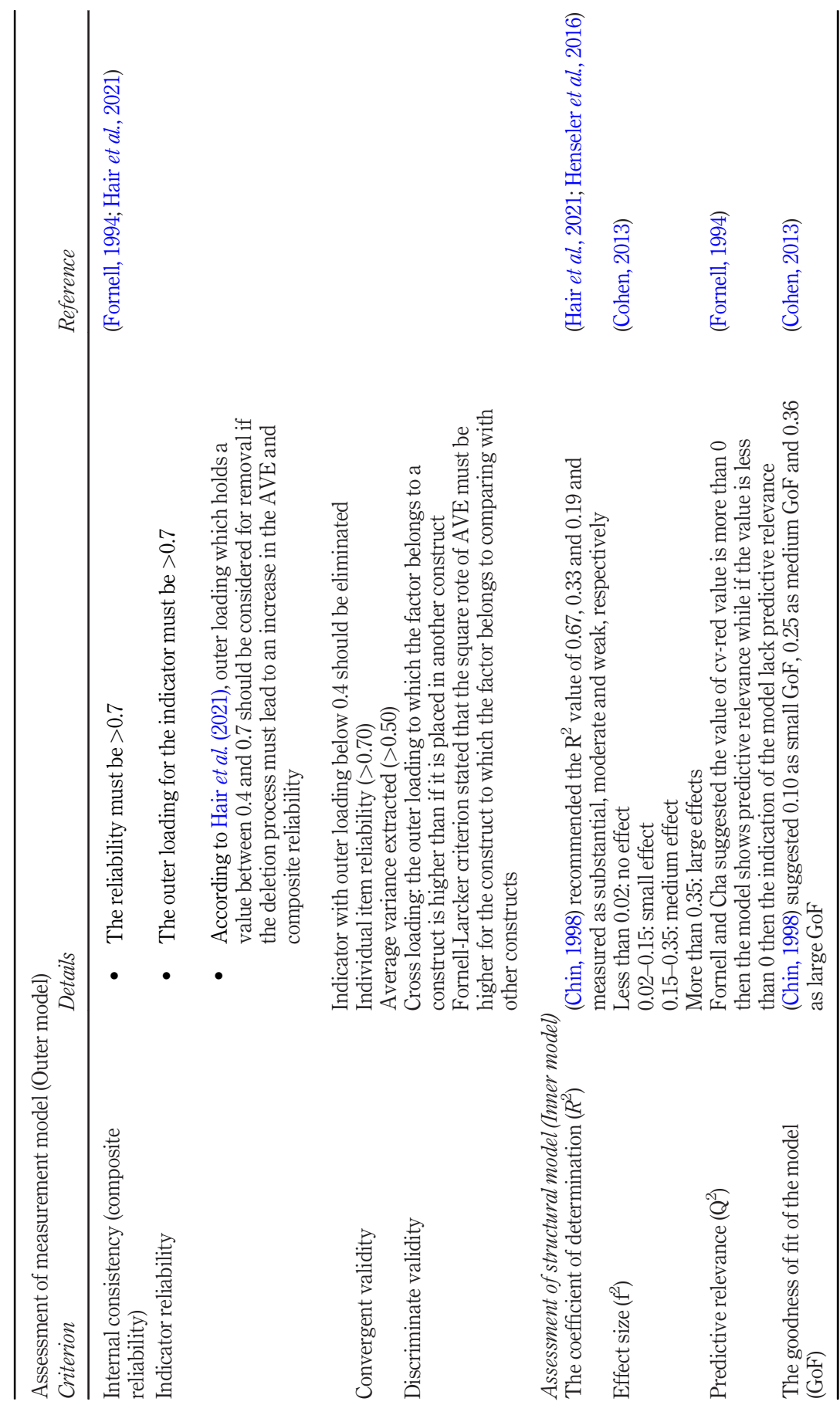
Table 1 presents the criteria used to assess the model for its components' measurement and structural models. These criteria must be achieved to consider the model's adequate representation to study the structural relationships among causes and effects of poor communication.

\section{Assessment of measurement model}

It is essential in developing PLS-SEM to check the reliability and validity of the measurement before generating the study results to ensure the methods and data are valid. The essential step is to check the internal consistency of the model; the correlation between the items is adequate for further analysis. The standard procedures consist of two conditions to be achieved: the first one is a model performance using individual item reliability and convergent validity and the second procedure is the discriminant validity, which is assessed after meeting the criterion in the first step. This involves a few iterations of the analysis by omitting factors that behold low loading (Henseler et al., 2016).

\subsection{Convergent validity (testing model's performance)}

Running of the PLS algorithm has to be associated with testing the measurement model to check its convergent validity and individual item reliability and those two criteria are related to each other (Henseler et al., 2015). Convergent validity is defined as the extent to which a measure relates positively to another measure of the same constructs (Hair et al., 2021). To check the convergent validity, three parameters have to be achieved average variance extracted (AVE) which has to be more than 0.5 , composite reliability (CR) which has to be more than 0.7 (Hair et al., 2021; Memon and Rahman, 2014; Nagapan, 2014) and internal consistency (Cronbach's alpha) which has to be more than 0.7 and in the case of exploratory research, 0.60 to 0.70 is acceptable (Hair et al., 2021; Mohamad et al., 2015). However, Peterson and Kim (2013) criticized that Cronbach's $\alpha$ is considered as a lower bound and inaccurate estimator of true reliability, nevertheless, CR is a more accurate estimator of internal reliability and (Wong, 2013), recommended not to use Cronbach's alpha to assess the internal consistency in PLS-SEM. The criterion of individual item reliability is described by Hair et al. (2021) which states that each item/manifest has to attain more than 0.5 and any factor that holds a value less than 0.5 has to be deleted and restart of the iteration process and model performance till achieving the minimum requirement described beforehand. In this study, the iteration process was performed until the validity and reliability criterions completed. Figure 5 shows the detailed parameters obtained from iteration 1.

Figure 5 shows the parameters of measurement model assessment, which is obtained from running the PLS algorithm for the first iteration. Tables 2 and 3 show the detailed and arranged factor loading for all the factors after running the first iteration.

Table 2 presents the final cause factors of poor communication and their symbols, which are used in the model. It also shows the factor loading generated from running algorithms for the first iteration.

Tables 2 and 3 show the factor loading resulted from running the first iteration. To decide which factor to delete the rule of thumb described by Hair et al. (2021) described that any manifest less than 0.4 is deleted and any factor more than 0.4 and less than 0.7 is deleted if there is a resultant of improvement in the $\mathrm{CR}$ and $\mathrm{AVE}$, therefore, an iterative check is performed to achieve the standards criterion. The following table presents the parameters of convergent validity assessment resulted from iteration 1 .

Table 4 presents the assessment of the convergent validity of the measurement model for the first iteration. It is shown that one construct (behavioral and cultural, $\mathrm{BC}$ ) has less than
Causes and effects of poor communication 
JFM

21,1

\section{2}

\section{Figure 5.}

Factor loading results of first iteration (Smart-PLS output)

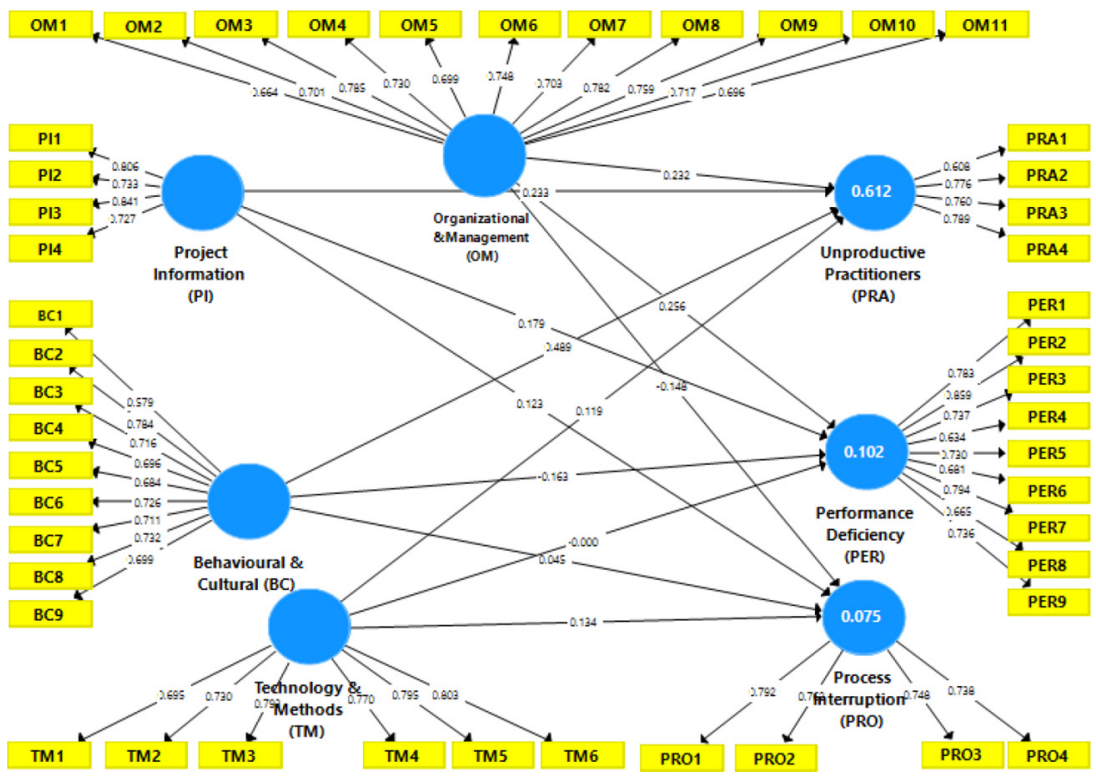

the criteria described in each parameter. This requires further repetition by deleting poorly measured manifests to achieving the standard values to develop a fit model as suggested by Nagapan (2014). In the second iteration, any factor that attains less than 0.6 outer loadings is deleted then checking the outcomes.

Table 5 shows the factor loading for causes factors resulted from iteration 2. After deleting one item, the factor loading improved to achieve the threshold value.

Table 6 shows the factor loading for effect factors, which is generated after running algorithms for iteration 2 .

Table 7 shows the convergent validity for the measurement model and shows that Cronbach's alpha, CR and AVE achieved all the standard requirements. The results show that the CR for all the constructs is more than 0.7 and the AVE is more than 0.5 and these results achieved the criteria of convergent validity.

\subsection{Discriminant validity}

After checking the model performance and achieving all the requirements stipulated by Hair et al. (2021), the following step in the assessment of the measurement model is to check the discriminant validity. Discriminant validity is defined as the degree to which the manifests differentiate among the constructs or measure different concepts by assessing the correlation among measures of potentially overlapping constructs (Hair et al., 2021; Wong, 2013). The discriminant validity is assessed using (Fornell and Larcker, 1981) criterion and cross-loadings (Henseler et al., 2015).

7.2.1 Cross loading. Cross loading assesses the discriminant validity by examining whether each manifest has a higher loading correlation with the IV in which the outer loading of each associated construct is more significant than all of its loading if applied on other constructs (Hair et al., 2021). 


\begin{tabular}{|c|c|c|c|c|}
\hline Group/constructs & Cause factors/items/manifests & Code & $\begin{array}{l}\text { Factor } \\
\text { loading }\end{array}$ & $\begin{array}{r}\text { Causes and effects } \\
\text { of poor }\end{array}$ \\
\hline \multirow{11}{*}{$\begin{array}{l}\text { Organizational } \\
\text { and management } \\
(\mathrm{OM})\end{array}$} & Lack of collaboration and representation & OM1 & 0.664 & \multirow{11}{*}{123} \\
\hline & Improper project planning & OM2 & 0.701 & \\
\hline & Infirm organizational communication structure & OM3 & 0.785 & \\
\hline & Lack of communication plan & OM4 & 0.73 & \\
\hline & Different level of technical skills & OM5 & 0.699 & \\
\hline & Poor project information management & OM6 & 0.748 & \\
\hline & Different level of education & OM7 & 0.703 & \\
\hline & Contractual barriers (restrictions) & OM8 & 0.782 & \\
\hline & Lack of formal execution of communication & OM9 & 0.759 & \\
\hline & Lack of communication skills & OM10 & 0.717 & \\
\hline & Frequent change of project contract & OM11 & 0.696 & \\
\hline \multirow{4}{*}{$\begin{array}{l}\text { Project } \\
\text { information (PI) }\end{array}$} & Lack of timely information & PI1 & 0.806 & \\
\hline & Poor project documentation & PI2 & 0.733 & \\
\hline & Inaccessibility of project information & PI3 & 0.841 & \\
\hline & Unavailability of crucial information & PI4 & 0.727 & \\
\hline \multirow{9}{*}{$\begin{array}{l}\text { Behavioral and } \\
\text { cultural (BC) }\end{array}$} & Individual habits & $\mathrm{BC} 1$ & 0.579 & \\
\hline & Lack of confidence & $\mathrm{BC} 2$ & 0.784 & \\
\hline & Fear to communicate & $\mathrm{BC} 3$ & 0.716 & \\
\hline & Negligence and casualness & $\mathrm{BC} 4$ & 0.696 & \\
\hline & Lack of ethics & BC5 & 0.684 & \\
\hline & Diversity of culture & $\mathrm{BC} 6$ & 0.726 & \\
\hline & Lack of understanding and acceptance & $\mathrm{BC} 7$ & 0.711 & \\
\hline & Language barrier & $\mathrm{BC} 8$ & 0.732 & \\
\hline & Usage of different terminologies & BC9 & 0.699 & \\
\hline \multirow{6}{*}{$\begin{array}{l}\text { Technology and } \\
\text { methods (TM) }\end{array}$} & Lack of appropriate communication medium & TM1 & 0.695 & \multirow{6}{*}{$\begin{array}{r}\text { Table } 2 . \\
\text { Factor loading for } \\
\text { cause factors } \\
\text { (iteration } 1)\end{array}$} \\
\hline & Improper communication channel & TM2 & 0.73 & \\
\hline & Lack of standardization of progress measurement & TM3 & 0.793 & \\
\hline & Lack of communication system and platform & TM4 & 0.77 & \\
\hline & Lack of interaction among different discipline & TM5 & 0.795 & \\
\hline & Improper introduction of new technology Ex. (BIM) & TM6 & 0.803 & \\
\hline
\end{tabular}

\begin{tabular}{|c|c|c|c|c|}
\hline Group/construct & Effect factors & Code & $\begin{array}{l}\text { Factor } \\
\text { loading }\end{array}$ & \\
\hline \multirow{4}{*}{$\begin{array}{l}\text { Unproductive } \\
\text { practitioners } \\
\text { (PRA) }\end{array}$} & Deteriorating relationship and cooperation & PRA1 & 0.608 & \\
\hline & High stress in the workplace & PRA2 & 0.776 & \\
\hline & Low level of satisfaction & PRA3 & 0.76 & \\
\hline & Demotivation & PRA4 & 0.789 & \\
\hline \multirow{9}{*}{$\begin{array}{l}\text { Performance } \\
\text { deficiency (PER) }\end{array}$} & Low productivity & PER1 & 0.783 & \\
\hline & Waste generation & PER2 & 0.859 & \\
\hline & Poor collaboration & PER3 & 0.737 & \\
\hline & Time overrun & PER4 & 0.634 & \\
\hline & Rework occurrence & PER5 & 0.73 & \\
\hline & Failure of the project & PER6 & 0.681 & \\
\hline & Safety standard violations & PER7 & 0.794 & \\
\hline & Dispute & PER8 & 0.665 & \\
\hline & Cost overrun & PER9 & 0.736 & \\
\hline & Redesign & PRO1 & 0.792 & Table 3. \\
\hline \multirow{3}{*}{ interruption (PRO) } & Misinterpreted technical information & $\mathrm{PRO} 2$ & 0.762 & Factor loading for \\
\hline & Frequent remedies in design and planning schedules & PRO3 & 0.748 & effect factors \\
\hline & Information overlapping & PRO4 & 0.738 & (iteration 1) \\
\hline
\end{tabular}


Table 8 shows the cross-loading analysis. It is demonstrated that the values generated in bold from cross-loading for each manifest have a higher value if it is placed in another construct/group and this indicated the discriminant validity concerning cross-loading is achieved.

7.2.2 Fornell and Larcker criterion. Fornell and Larcker's (1981) criterion is also used to assess the discriminant validity of the model by comparing the square root of AVE with

Table 4.
Convergent validity of measurement model (iteration 1)

\begin{tabular}{llccc}
\hline Variables & Constructs/Group & $\alpha(\geq 0.7)$ & CR $(\geq 0.7)$ & AVE $(\geq 0.5)$ \\
\hline Causes & Organizational and management (OM) & 0.911 & 0.925 & 0.528 \\
& Project information (PI) & 0.782 & 0.859 & 0.605 \\
& Behavioral and cultural (BC) & 0.872 & 0.898 & $\mathbf{0 . 4 9 7}$ \\
& Technology and methods (TM) & 0.862 & 0.894 & 0.586 \\
Effects & Unproductive practitioners (PRA) & 0.717 & 0.825 & 0.543 \\
& Performance deficiency (PER) & 0.898 & 0.915 & 0.545 \\
& Process interruption (PRO) & 0.763 & 0.846 & 0.578
\end{tabular}

Note: *Indicates the value is less than the threshold criterion

\begin{tabular}{llcc}
\hline Group/constructs & Cause factors/items/manifests & Code & Factor loading \\
\hline Organizational and & Lack of collaboration and representation & OM1 & 0.664 \\
management (OM) & Improper project planning & OM2 & 0.701 \\
& Infirm organizational communication structure & OM3 & 0.785 \\
& Lack of communication plan & OM4 & 0.73 \\
& Different level of technical skills & OM5 & 0.698 \\
& Poor project information management & OM6 & 0.747 \\
& Different level of education & OM7 & 0.703 \\
& Contractual barriers (restrictions) & OM8 & 0.782 \\
& Lack of formal execution of communication & OM9 & 0.759 \\
& Lack of communication skills & OM10 & 0.717 \\
Project information (PI) & OM11 & 0.696 \\
& Lack of timely information contract & PI1 & 0.806 \\
& Poor project documentation & PI2 & 0.733 \\
Inaccessibility of project information & PI3 & 0.841 \\
Behavioral and cultural & PI4 & 0.727 \\
(BC) & Unavailability of crucial information & BC1 & Deleted \\
& Lack of confidence & BC2 & 0.793 \\
& Fear to communicate & BC3 & 0.707 \\
& Negligence and casualness & BC4 & 0.717 \\
& Lack of ethics & BC5 & 0.711 \\
& Diversity of culture & BC6 & 0.722 \\
& Lack of understanding and acceptance & BC7 & 0.705 \\
& Language barrier & BC8 & 0.753 \\
Technology and & Usage of different terminologies & BC9 & 0.68 \\
methods (TM) & Lack of appropriate communication medium & TM1 & 0.695 \\
& Improper communication channel & TM2 & 0.73 \\
& Lack of standardization of progress measurement & TM3 & 0.793 \\
& Lack of communication system and platform & TM4 & 0.77 \\
& Lack of interaction among different discipline & TM5 & 0.795 \\
& Improper introduction of new technology Ex. (BIM) & TM6 & 0.803 \\
\hline & & & \\
& & & \\
& & &
\end{tabular}

Table 5.

Factor loading for cause factors (iteration 2) 


\begin{tabular}{|c|c|c|c|c|}
\hline Group/Construct & Effect factors & Code & Factor loading & 4. \\
\hline \multirow{4}{*}{$\begin{array}{l}\text { Unproductive } \\
\text { practitioners (PRA) }\end{array}$} & Deteriorating relationship and cooperation & PRA1 & 0.610 & \multirow{4}{*}{ communication } \\
\hline & High stress in the workplace & PRA2 & 0.777 & \\
\hline & Low level of satisfaction & PRA3 & 0.758 & \\
\hline & Demotivation & PRA4 & 0.788 & \\
\hline \multirow{9}{*}{$\begin{array}{l}\text { Performance deficiency } \\
\text { (PER) }\end{array}$} & Low productivity & PER1 & 0.783 & \multirow{9}{*}{125} \\
\hline & Waste generation & PER2 & 0.859 & \\
\hline & Poor collaboration & PER3 & 0.737 & \\
\hline & Time overrun & PER4 & 0.635 & \\
\hline & Rework occurrence & PER5 & 0.731 & \\
\hline & Failure of the project & PER6 & 0.681 & \\
\hline & Safety standard violations & PER7 & 0.793 & \\
\hline & Dispute & PER8 & 0.667 & \\
\hline & Cost overrun & PER9 & 0.736 & \\
\hline Process interruption & Redesign & PRO1 & 0.791 & \multirow{4}{*}{$\begin{array}{r}\text { Factor loading for } \\
\text { effect factors } \\
\text { (iteration 2) }\end{array}$} \\
\hline \multirow{3}{*}{ (PRO) } & Misinterpretation of technical information & PRO2 & 0.762 & \\
\hline & Frequent remedies in design and planning schedules & PRO3 & 0.748 & \\
\hline & Information overlapping & PRO4 & 0.739 & \\
\hline
\end{tabular}

\begin{tabular}{llccc}
\hline Categories & Constructs/Group & $\alpha(\geq 0.7)$ & CR $(\geq 0.7)$ & AVE ( $\geq 0.5)$ \\
\hline Causes & Organizational and management (OM) & 0.911 & 0.925 & 0.528 \\
& Project information (PI) & 0.782 & 0.859 & 0.605 \\
& Behavioral and cultural (BC) & 0.87 & 0.898 & 0.525 \\
\multirow{4}{*}{ Effects } & Technology and methods (TM) & 0.862 & 0.894 & 0.586 \\
& Unproductive practitioners (PRA) & 0.717 & 0.825 & 0.543 \\
& Performance deficiency (PER) & 0.898 & 0.915 & 0.546 \\
& Process interruption (PRO) & 0.763 & 0.845 & 0.578
\end{tabular}

Notes: *Indicates the value is less than the threshold value

Table 7.

Convergent validity of measurement model (iteration 2)

latent variable correlations in which the square root construct should be higher than its highest correlation with any other constructs (Hair et al., 2021). The criterion of Fornell and Larcker stated that the latent variable must explain a better variance of its indicator than the variance of other latent variables (Ab Hamid et al., 2017). Fornell and Larcker is considered a significant approach for assessing the discriminant validity of PLS-SEM and it works by comparing the square root of the AVE with the independent variable correlations (Hair et al., 2021). The latent variable correlation generated from running the PLS algorithm is shown in Table 9 .

Table 9 demonstrates the correlations of the latent variables and it is shown that the root square of AVE at the diagonal matrix for each variable is higher than the non-diagonal values, which indicated in boldface and this is satisfied the criterion of Fornell and Larcker, therefore, discriminate validity is fulfilled and the following step is to discuss the outcomes of measurement model assessment.

\subsection{Discussion of measurement model results}

The measurement model has been previously verified and assessed by satisfying all the prerequisite criteria prescribed in Table 1 by running two subsequent iterations of algorithms. The first step achieved is convergent validity, which is the degree to which 


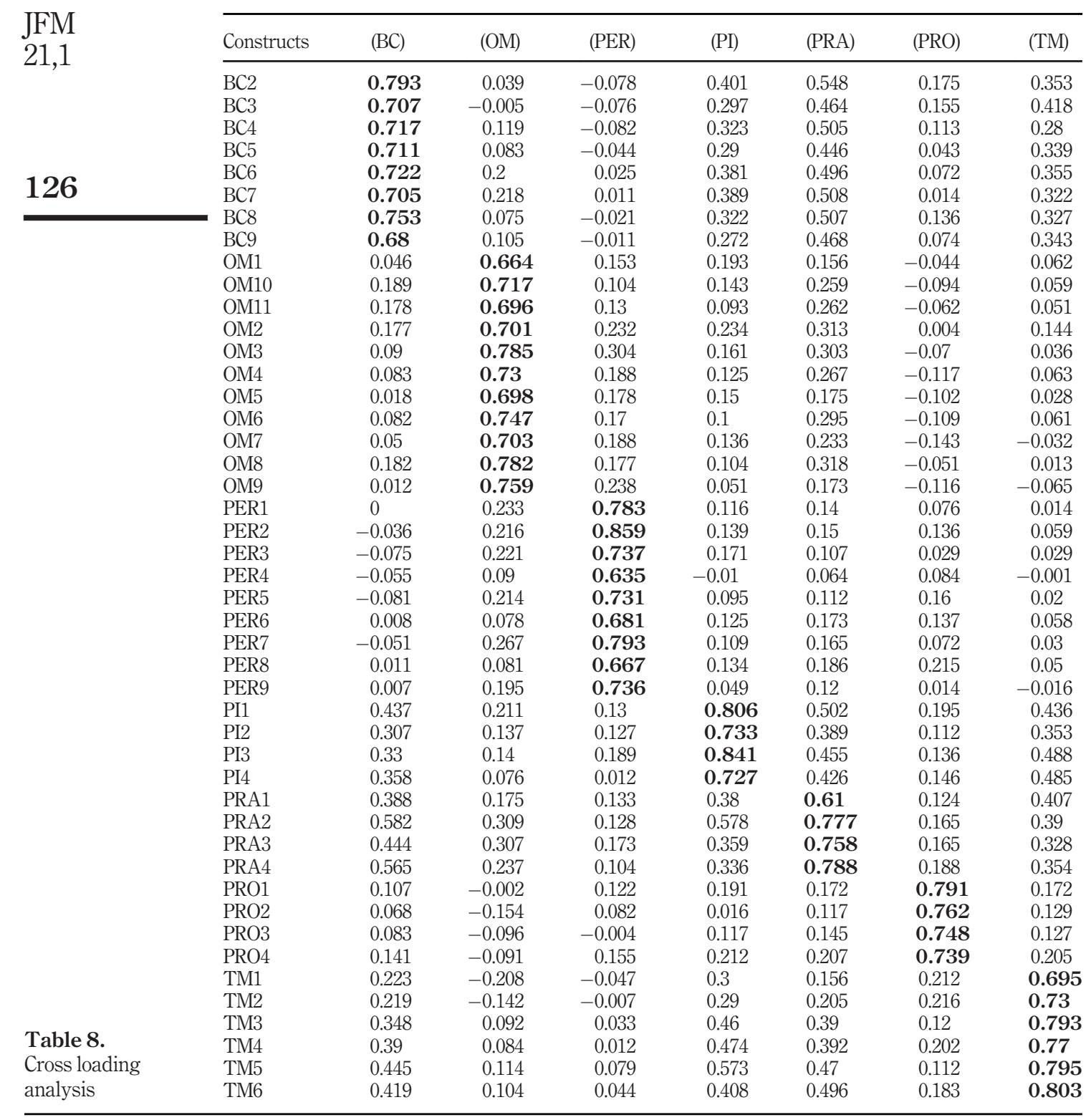

factors are related to measuring their corresponding constructs. From the data described in Tables 5 and 6 , the factor loadings are more than 0.5 and vary among different items.

The constructs under causative factors are organization and management, PI, BC, technologies and methods. For the first construct organizational and management $(\mathrm{OM})$, the most loaded factor is infirm organizational communication structure with 0.785 loadings 
and that exhibited the significance of this factor in comparison to other adjacent factors, Causes and effects which duly proved the lack of awareness toward managing communication in construction projects. Regarding the second causative construct, which is PI, the inaccessibility of PI factor with 0.841 loadings is the most important factor and that interprets higher contribution to cause poor communication. The third causative construct is $\mathrm{BC}$ in which a lack of confidence factor (0.793) has the most loading factor under the construct and this explains the gap of information exchange in the project space. Regarding the fourth construct (technology and methods, TM), the most weighted factor is the improper introduction of new technology (0.803) and that described the issue of implementing newly added technologies, such as BIM in its different dimensions as a method of managing and executing the project.

Table 6 presented the factor loading for effect factors and that includes three constructed named practitioners, performance and process. From the results, factors under practitioners vary in the loading weight and demotivation weighted the most factor with 0.788 and that signified the effect of improper communication and causing demotivation to the workforce. However, for the performance construct the most prevalent factor is waste generation with 0.859 and that was earlier proven by Nagapan (2014) which considered poor communications a leading factor to cause waste generation. Regarding the processes, the most weighed factor is a redesign with 0.791 and that critically occurs during the early stage of the project. Referring to Table 7 , all the measures of convergent validity achieved the threshold criteria in which Cronbach's $\alpha$ is more than 0.7 , CR is more than 0.7 and AVE is more than 0.5 , hence the model satisfied the convergent validity.

The second step is to check the discriminant, which is intended to ensure the construct has the most substantial relationship with its own manifests in comparison with other constructs. Cross loading and Fornell and Larcker standards are used to assess the discriminant validity, which collectively proved acceptable and valid discriminant.

In conclusion, the measurement model is assessed for its validity and reliability and it can be concluded that the iteration process applied and modifications have positively improved the model performance. All the weak manifests were deleted and after iteration two the required threshold values were accomplished for all the criteria, therefore, the measurement model is assessed and further assessment of the structural model followingly is carried out.

\section{Assessment of structural model}

After confirming the reliability and validity of the outer model, the following step is to assess the inner model by evaluating the model's predictive capabilities and relationships among constructs. The structural model is defined as the networked relationships, which

\begin{tabular}{lcclllll}
\hline Constructs & BC & OM & PER & PRA & PRO & PI & TM \\
\hline (BC) & $\mathbf{0 . 7 2 4}$ & & & & & & \\
(OM) & 0.142 & $\mathbf{0 . 7 2 7}$ & & & & & \\
(PER) & -0.049 & 0.265 & $\mathbf{0 . 7 3 9}$ & & & & \\
(PRA) & 0.682 & 0.354 & 0.18 & $\mathbf{0 . 7 3 7}$ & & & \\
(PRO) & 0.138 & -0.111 & 0.127 & 0.219 & $\mathbf{0 . 7 6}$ & & \\
(PI) & 0.464 & 0.186 & 0.151 & 0.573 & 0.192 & $\mathbf{0 . 7 7 8}$ & \\
(TM) & 0.471 & 0.054 & 0.037 & 0.499 & 0.217 & 0.567 & $\mathbf{0 . 7 6 5}$
\end{tabular}

Notes: The values in the boldface are the square root of AVE 
JFM

21,1

128

connected the hypothetical model whereby the purpose of this assessment is to examine the relationship among dependent and IV (Hair et al., 2021) and the significant steps to assess the structural model are presented in Figure 6.

Figure 6 shows the steps required to assess the structural model, which sequentially starts by testing the predefined hypothesis then checking the ability of IV in measuring the DV using the coefficient of determination. Following that, assessing the effect size and assessing the predictive relevance by evaluating the confidence of the model and finally checking the goodness of fit (GoF) for the model to measure the overall model's performance.

\subsection{Hypothesis testing}

Hypothesis testing is performed to confirm the significance values statistically (Yuan, 2012) and check the hypothesized relationship among constructs in which the causes (IV) have a significant impact on the effect factors (DV). To determine whether the relationship is significant or not, both $t$-values and $p$-values are used as a cutoff value whereby $T$-value has to be more than 1.96 or $P$-value has to be less than 0.05 (Hair et al., 2021).

Table 10 shows the results of hypothesis testing after running bootstrapping in SmartPLS3. The bootstrapping was run using subsamples of 5000 to achieve maximum stability of the results (Hair et al., 2021) According to Table 10, eight hypotheses out of twelve have a significant relationship with the pre-assigned path model. These include the groups/ constructs, which obtained a T-value higher than 1.96 and a $p$-value less than 0.05 . Those IV (constructs/groups) which have no significant value on the DV is explained by the data obtained from respondents have no significant power to find the dependency level. Therefore, more investigation is required to improve the model. The generated $t$-values for the structural model are shown in Figure 7.

Figure 7 shows the $t$-values generated from running bootstrapping for the model. It shows that any $t$-values that exhibit less than 1.96 are considered insignificant.

\subsection{Coefficient of determination $\left(R^{2}\right)$}

The coefficient of determination is defined as a statistical criterion to measure how the regression predictions are accurately fit for the data. In PLS-SEM, it represents the extent of variation in the dependent variables, which can describe one or more predictor variables (Hair et al., 2021). In other words, it measures the ability of IV in measuring dependent variables. As a cut-off value, Falk and Miller (1992) suggested an $R^{2}$ value of 0.10 as a minimum acceptable value for the model, nevertheless, Chin (1998) recommended the $R^{2}$

Figure 6.

Assessment steps of structural model (adapted from: (Hair et al., 2021))

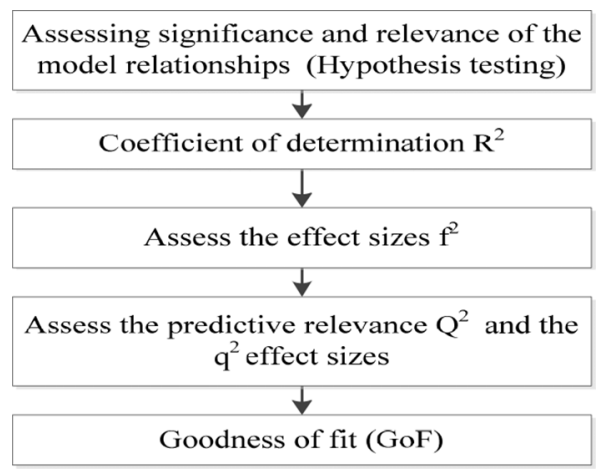




\begin{tabular}{|c|c|c|c|c|c|}
\hline Hypothesis & Relationships & $t$-values & $p$-values & Decision & or \\
\hline$H 1$ & $\begin{array}{l}\text { Behavioral and cultural (BC) } \rightarrow \text { Performance deficiency } \\
\text { (PER) }\end{array}$ & 2.412 & 0.016 & Supported & communication \\
\hline$H 2$ & $\begin{array}{l}\text { Behavioral and cultural }(\mathrm{BC}) \rightarrow \text { Unproductive } \\
\text { practitioners }(\mathrm{PRA})\end{array}$ & 10.071 & 0.000 & Supported & \\
\hline H3 & $\begin{array}{l}\text { Behavioral and cultural }(\mathrm{BC}) \rightarrow \text { Process interruption } \\
(\mathrm{PRO})\end{array}$ & 0.543 & 0.587 & Not supported & 129 \\
\hline$H 4$ & $\begin{array}{l}\text { Organizational and management }(\mathrm{OM}) \rightarrow \text { Performance } \\
\text { deficiency (PER) }\end{array}$ & 4.688 & 0.000 & Supported & \\
\hline H5 & $\begin{array}{l}\text { Organizational and management }(\mathrm{OM}) \rightarrow \\
\text { Unproductive practitioners (PRA) }\end{array}$ & 6.303 & 0.000 & Supported & \\
\hline$H 6$ & $\begin{array}{l}\text { Organizational and management }(\mathrm{OM}) \rightarrow \text { Process } \\
\text { interruption (PRO) }\end{array}$ & 2.153 & 0.032 & Supported & \\
\hline$H 7$ & $\begin{array}{l}\text { Project information (PI) } \rightarrow \text { Performance deficiency } \\
\text { (PER) }\end{array}$ & 2.150 & 0.032 & Supported & \\
\hline H8 & $\begin{array}{l}\text { Project information }(\mathrm{PI}) \rightarrow \text { Unproductive practitioners } \\
(\mathrm{PRA})\end{array}$ & 4.516 & 0.000 & Supported & \\
\hline H9 & Project information (PI) $\rightarrow$ Process interruption (PRO) & 1.310 & 0.190 & Not supported & \\
\hline H1O & $\begin{array}{l}\text { Technology and methods (TM) } \rightarrow \text { Performance } \\
\text { deficiency (PER) }\end{array}$ & 0.003 & 0.997 & Not supported & \\
\hline$H 11$ & $\begin{array}{l}\text { Technology and methods (TM) } \rightarrow \text { Unproductive } \\
\text { PRActitioners (PRA) }\end{array}$ & 2.443 & 0.015 & Supported & Tab \\
\hline$H 12$ & $\begin{array}{l}\text { Technology and methods (TM) } \rightarrow \text { Process interruption } \\
\text { (PRO) }\end{array}$ & 1.520 & 0.129 & Not supported & $\begin{array}{r}\text { Model hypothesis } \\
\text { testing }\end{array}$ \\
\hline
\end{tabular}

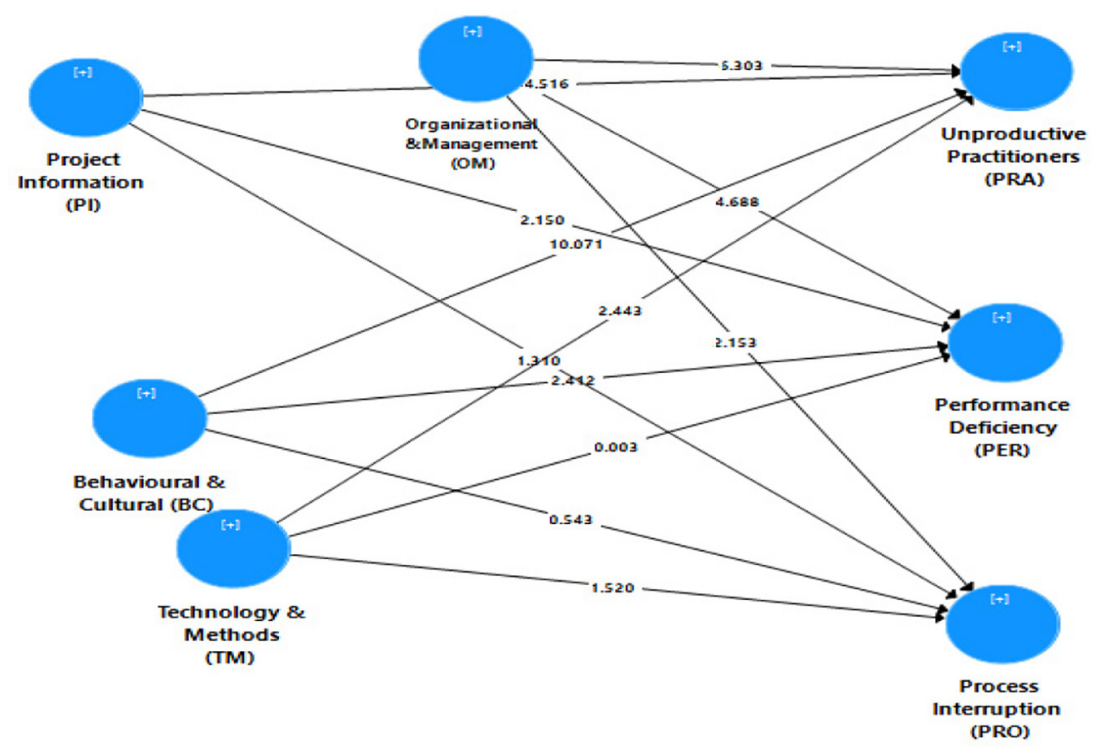

Figure 7. Structural model $t$-values generated from bootstrapping (Smart-PLS output) 
JFM

21,1

Table 11.

$\mathrm{R}$-square of the endogenous latent variables value of above 0.67 considered substantial while values ranging between 0.67 and 0.33 are moderate and values less than 0.19 are weak.

Table 11 shows the results of the R-square for the model. It is shown that the constructs PER and PRO have weak R-square while PRA is moderate, henceforth, the results explain that the power in representing the groups is satisfactory for PRA and shows the higher ability of IV in measuring DVbut weak for PER and PRO and that is explained due to the less complexity of the model in which more predictive latent variable increases the coefficient of determination (Tenenhaus et al., 2005; Yuan, 2012).

\subsection{Effect size}

Effect size $\left(\mathrm{f}^{2}\right)$ is an indicator of the degree of the relative effect of exogenous latent variables on an endogenous latent variable by the mean of change in $R^{2}$ and it is calculated as the increase of R-squared of the latent variable in which the path is connected (Chin, 1998). According to the guidelines introduced by Cohen (2013) the effect size can be evaluated if the value of $\mathrm{f}^{2}$ is less than 0.02 is then is considered as no effect size, from 0.02 to 0.15 is a small effect, from 0.15 to 0.35 medium effect size and more than 0.35 is a large effect of the endogenous latent variables.

Table 12 shows the $\mathrm{f}^{2}$ values after running the algorithm for the model. The results show different size effects of the exogenous variables on the endogenous variables.

\subsection{Model's Predictive relevance $\left(Q^{2}\right)$}

Predictive relevance $\left(Q^{2}\right)$ is an indicator of the model's ability to predict values from the data. It is obtained using the approach of blindfolding, which is defined as the sample reuse technique that deletes every distance (D) of the data points in the endogenous construct indicators and estimates the parameters with the remaining data point besides the

\begin{tabular}{lcr}
\hline Dependent variables (DV) & R-square & Remarks \\
\hline Performance deficiency (PER) & 0.103 & Weak \\
Unproductive practitioners (PRA) & 0.608 & Moderate \\
Process interruption (PRO) & 0.075 & Weak \\
\hline
\end{tabular}

\begin{tabular}{|c|c|c|c|}
\hline Constructs & Latent variables & f-squared & $\begin{array}{l}\text { Effect size } \\
\text { (Cohen, 1988) }\end{array}$ \\
\hline \multirow[t]{3}{*}{ Behavioral and cultural (BC) } & Performance deficiency (PER) & 0.023 & Small effect \\
\hline & unproductive practitioners (PRA) & 0.420 & Large effect \\
\hline & process interruption (PRO) & 0.001 & No effect \\
\hline \multirow[t]{3}{*}{ Organizational and management $(\mathrm{OM})$} & Performance deficiency (PER) & 0.069 & Small effect \\
\hline & Unproductive practitioners (PRA) & 0.135 & Medium effect \\
\hline & Process interruption (PRO) & 0.022 & Small effect \\
\hline \multirow{3}{*}{ Project information (PI) } & Performance deficiency (PER) & 0.023 & Small effect \\
\hline & Unproductive practitioners (PRA) & 0.086 & Small effect \\
\hline & Process interruption (PRO) & 0.010 & No effect \\
\hline \multirow[t]{3}{*}{ Technology and methods (TM) } & Performance deficiency (PER) & 0.000 & No effect \\
\hline & Unproductive practitioners (PRA) & 0.012 & No effect \\
\hline & Process interruption (PRO) & 0.026 & Small effect \\
\hline
\end{tabular}

Table 12.

Effect size $\left(\mathrm{f}^{2}\right)$ for the model 
blindfolding procedure is only applied to latent constructs, which have a reflective measurement (Hair et al., 2021; Henseler et al., 2016). Q ${ }^{2}$ is a form of statistical validation for confidence in the model (Yuan, 2012). The recommended omission distance is between 5 and 12 (Chin, 1998; Hair et al., 2021). In smart-PLS two values are calculated, which are crossvalidated redundancy (cv-red) and cross-validated communality (cv-comm); however, for the assessment purpose, the cv-red is used to evaluate the model. According to the recommendation by Fornell and Larcker (1981) if the value of cv-red value is more than 0 then the model shows predictive relevance while if the value is less than 0 then the indication of the model lack predictive relevance.

Figure 8 shows the results obtained from blindfolding predictive relevance. The values are arranged accordingly in Table 13.

Table 13 shows the predictive relevance values for dependent variables. It is shown that all the values are more than 0 . Therefore, this indicates the model has satisfactory predictive relevance and is fit to predict endogenous variables.

\subsection{Goodness of fit}

The GoF is an index used to measure the geometric mean of the average communality and average coefficient of determination for all endogenous constructs (Tenenhaus et al., 2005). It is also used to evaluate the reflective indicators and is considered as a factor for assessing

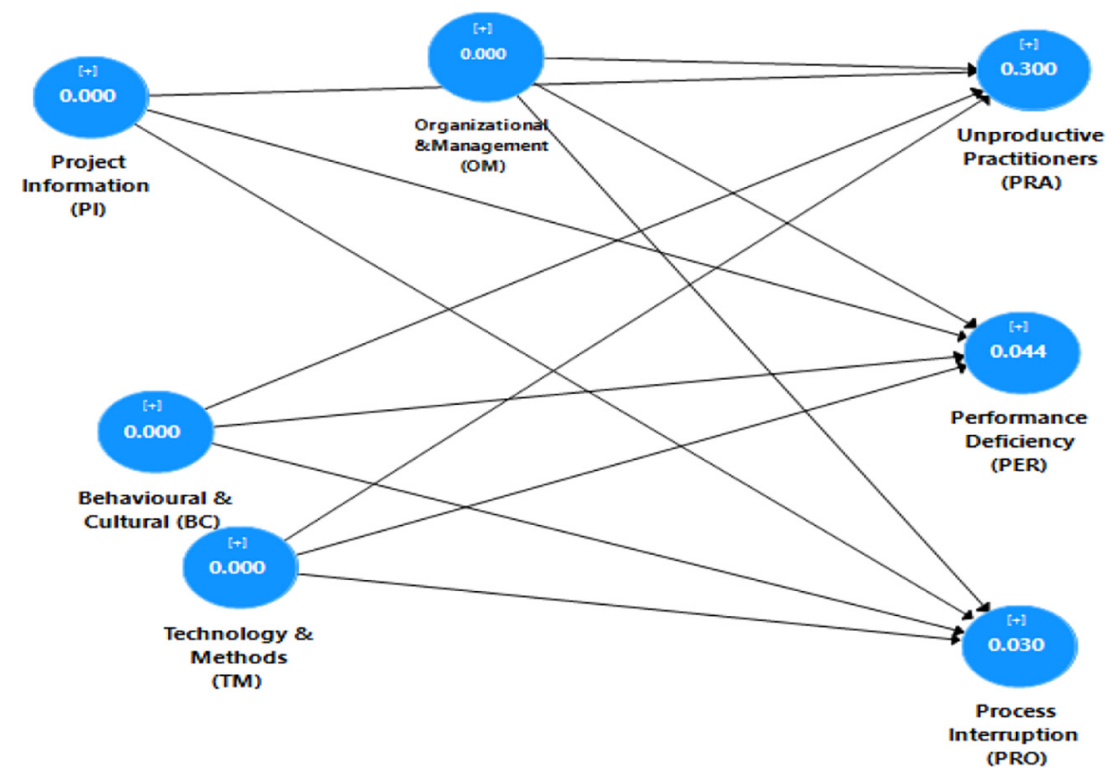

Dependent variables
Causes and effects of poor communication

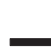

\section{.}


JFM

21,1

the global fit and validity of the model. The primary purpose of determining GoF is to test whether to account for the study model for both components measurement and structural model of its overall performance (Chin, 1998; Tenenhaus et al., 2005; Vinzi et al., 2010; Yuan, 2012). There have been many criticisms on the cut-off value of GoF, (Wetzels et al., 2009) suggested the cut-off value of GoF to be 0.5 for the commonality. However, Cohen (Cohen, 2013) suggested 0.10 as small GoF, 0.25 as medium GOF and 0.36 as large GOF. Calculation of $\mathrm{GoF}$ is performed manually using the following formula (Coughlan, 2008):

$$
G o F=\sqrt{\left(\overline{R^{2}} \times \overline{A V E}\right.}
$$

Referring to Table 7 and Table 11, the overall average value of AVE is 0.56 and the average value of $R^{2}$ is 0.26 , then GoF is calculated as follows:

$$
G o F=\sqrt{(0.26 \times 0.56=0.38}
$$

To decide upon the evaluation of GoF value, the standard criteria suggested by Wetzels et al. (2009) stated that GoF is considered. No fit if the value of GoF is less than 0.1, between 0.1 and 0.25 is a small fit, 0.25 to 0.36 is medium fit and more than 0.36 is considered an ample fit. According to the value calculated using the $\mathrm{GoF}$ formula (0.38) and the criteria suggested by Wetzels et al. (2009). It can be concluded that the GoF for the model is large enough to be considered to obtain sufficient global PLS model validity.

After finalizing all the assessments of the structural model, the developed hypothetical model is presented and then the relationship among cause-and-effect factors of poor communication in the construction industry, hence after performing the structural model assessment. As a result, the final PLS-SEM model of the relationship between cause-andeffect factors of poor communication in the construction industry is presented in Figure 9.

Figure 9 shows the final PLS-SEM for the relationship between the causes and effects of poor communication in the construction industry. From the figure, the indication of relationship strength is interpreted by the path coefficient, which shows the extent of the relationship between causes (IVs) and effect (DVs). It is indicated that OM has a strong relationship with PER and weak relation with process interruption (PRO) and the path coefficient values explain this.

\section{Discussion of structural model results}

The model developed intended to study the relationships among causes and effects, which considered effects are the subsequent outcome of the causes. Thus, a comprehensive investigation of the collective relationships is required to take preventative measures of the causes at the early stages of the project. This section explains the outcomes of structural model assessments.

According to Table 10, the outcomes show three of the hypothesized paths are not significant, which proves no relationship between those constructs. To explain further on the relationships among causes and effects, the following discussions are outlined:

- The causative factors of poor communication in construction under the construct named $\mathrm{BC}$ exhibited relationship with the effect construct named performance and practitioners, however, it did not support any relationship with the process, which relatively provides logical justification of the outcome, hence behavioral factors, such as individual habits can impact on the performance and the demotivation of practitioners. 


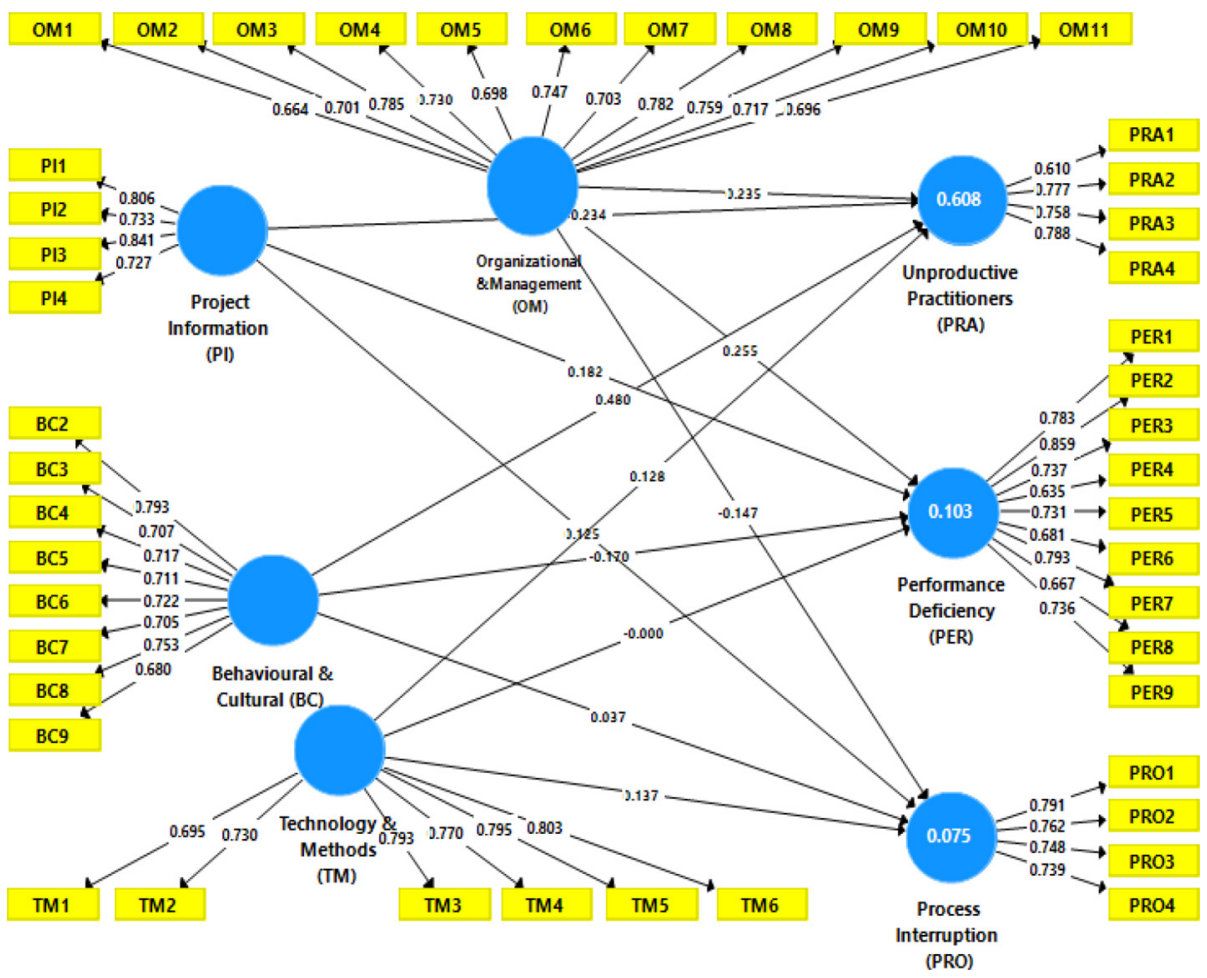

Causes and effects of poor communication

133

Figure 9. Final PLS-SEM model of poor communication

- OM causative factors poor communication have a significant relationship to the effect's underlining performance, practitioners and process, which explains the importance of management and organizational standpoints in the involvement of the project throughout the lifecycle.

- The construct PI has a noticeable relationship to cause deterioration of performance, practitioners' productivity but is slightly significant to cause a process breakdown.

- TM have a relationship with practitioners and process, however, no detection of significance in relation to the performance.

Regarding the effect size, which is an indicator of the relative effect of causes factors on the effect's factors, from Table 12, the following results are produced:

- $\mathrm{BC}$ related causes factors have a small impact on causing PER, however, it has a large impact on producing unproductive practitioners (PRA) and no impact on the process (PRO).

- The causes under OM have a small effect on PER and PRO and medium impact in producing PRA.

- The cause factors of poor communication related to PI have a small effect on PER and creating PRA but no effect on PRO.

- The cause factors of poor communication, which are related to TM do not affect the PER and PRA, nevertheless, it shows a small effect on PRO. 
Referring to Figure 9, which shows the final model of cause-and-effect factors of poor communication in construction. It indicates the relationship strength among causes (IVs) and effects (DVs) by the path coefficient values. From the figure, it is shown that causes factors under OM have the most substantial relationship with performance deficiency. Regarding the causes under $\mathrm{BC}$, the most substantial relationship exhibited with PRArelated effects. Similarly, for the causes under the PI corresponding causes shows the strongest relationship with PRA. However, the causes under technology and method show the strongest relationship with process deficiency.

\section{Model outcome interpretation and verification}

Although the SEM has been previously assessed and verified statistically for its measurement and structural components. There is still one more process to be carried out to accept the model as a representation in the construction industry. This part introduces the interpretation and verification process of the model through the participation of construction experts. Interviews were carried out to share their understandings and opinions on the model's applicability in the construction projects setting. The method is carried out by dividing the outcomes of the model into three main parts, which the first part includes the measurement model by ranking the factors based on their importance while the second part is evaluating the effects of the relationship among causes and effects. The final part is to investigate the possibility of applying the model in construction projects.

\subsection{Model interpretation}

This part aims to interpret the model of poor communication cause and effect factors with the reference to previous findings in previous sections. The purpose of interpreting the model is to produce understandable outcomes for participants. Table 14 shows the ranking of the factors based on the factor loading produced by the final iteration. The model comprises four groups called IV (causes) and three groups of DV (effects).

Table 14 shows the ranking of causative factors of poor communication based on the loading generated from SEM. The ranking displayed is based on constructs.

Table 15 shows the effect factors ranking based on the individual factor loading for each construct. It is shown that demotivation ranks first under the unproductive practitioner's group, waste generation underperformance and redesign under process corresponding effect. The second part of the outcome is the relationship analysis among causes (IVs) and effect (DVs) groups, which is explained by the path coefficient illustrated in previous sections in the final model. Table 16 shows the relationship among causes and effects of poor communication in the construction industry.

Table 16 shows the relationships among cause-and-effect factors of poor communication using the path coefficients illustrated in the final model presented earlier. The following part is to verify these findings by involving construction experts.

\subsection{Model verification process}

The data obtained from the model were verified by selected experts. The purpose of carrying model verification is to relate the data obtained statistically with the construction project setting. The expert attributes have been defined as a person whose age is more than 30 , worked in construction for more than 10 years and who is positioned in a managerial post. Semi-structured questions interviews were conducted alongside with questionnaires to verify the outcomes. A total of 14 experts participated in the model verification process. The first section of the question is about their background, the second part evaluating the factor 


\begin{tabular}{|c|c|c|c|c|}
\hline Group/Constructs & Cause factors/items/manifests & Code & Ranking & of poor \\
\hline \multirow[t]{11}{*}{ Organizational and management } & Infirm organizational communication structure & OM3 & 1 & communication \\
\hline & Contractual barriers (restrictions) & OM8 & 2 & \\
\hline & Lack of formal execution of communication & OM9 & 3 & \\
\hline & Poor project information management & OM6 & 4 & \\
\hline & Lack of communication plan & OM4 & 5 & \\
\hline & Lack of communication skills & OM10 & 6 & 135 \\
\hline & Different level of education & OM7 & 7 & \\
\hline & Improper project planning & OM2 & 8 & \\
\hline & Different level of technical skills & OM5 & 9 & \\
\hline & Frequent change of project contract & OM11 & 10 & \\
\hline & Lack of collaboration and representation & OM1 & 11 & \\
\hline \multirow{4}{*}{ Project information } & Inaccessibility of project information & PI3 & 1 & \\
\hline & Lack of timely information & PI1 & 2 & \\
\hline & Poor project documentation & PI2 & 3 & \\
\hline & Unavailability of crucial information & PI4 & 4 & \\
\hline \multirow[t]{8}{*}{ Behavioral and cultural } & Lack of confidence & $\mathrm{BC} 2$ & 1 & \\
\hline & Language barrier & $\mathrm{BC} 8$ & 2 & \\
\hline & Diversity of culture & $\mathrm{BC} 6$ & 3 & \\
\hline & Negligence and casualness & $\mathrm{BC} 4$ & 4 & \\
\hline & Lack of ethics & BC5 & 5 & \\
\hline & Fear to communicate & $\mathrm{BC} 3$ & 6 & \\
\hline & Lack of understanding and acceptance & $\mathrm{BC} 7$ & 7 & \\
\hline & Usage of different terminologies & BC9 & 8 & Table \\
\hline \multirow[t]{6}{*}{ Technology and methods } & Improper introduction of new technology & TM6 & 1 & Ranking of \\
\hline & Lack of interaction among different discipline & TM5 & 2 & Ranking of cau \\
\hline & Lack of standardization of progress measurement & TM3 & 3 & factors of poor \\
\hline & Lack of communication system and platform & TM4 & 4 & communication \\
\hline & Improper communication channel & TM2 & 5 & based on factor \\
\hline & Lack of appropriate communication medium & TM1 & 6 & loadings \\
\hline
\end{tabular}

\begin{tabular}{|c|c|c|c|c|}
\hline Group/Construct & Effect factors & Code & Ranking & \\
\hline \multirow[t]{4}{*}{ Unproductive practitioners } & Demotivation & PRA4 & 1 & \\
\hline & High stress in the workplace & PRA2 & 2 & \\
\hline & Low level of satisfaction & PRA3 & 3 & \\
\hline & Deteriorating relationship and cooperation & PRA1 & 4 & \\
\hline \multirow{9}{*}{ Performance deficiency } & Waste generation & PER2 & 1 & \\
\hline & Safety standard violations & PER7 & 2 & \\
\hline & Low productivity & PER1 & 3 & \\
\hline & Poor collaboration & PER3 & 4 & \\
\hline & Cost overrun & PER9 & 5 & \\
\hline & Rework occurrence & PER5 & 6 & \\
\hline & Failure of the project & PER6 & 7 & \\
\hline & Dispute & PER8 & 8 & Ranking of effect \\
\hline & Time overrun & PER4 & 9 & Ranking of effect \\
\hline \multirow[t]{4}{*}{ Process interruption } & Redesign & PRO1 & 1 & factors of poor \\
\hline & Misinterpreted technical information & $\mathrm{PRO} 2$ & 2 & communication \\
\hline & Frequent remedies in design and planning schedules & PRO3 & 3 & based on factor \\
\hline & Information overlapping & $\mathrm{PRO} 4$ & 4 & loadings \\
\hline
\end{tabular}


JFM

21,1

ranking obtained from the measurement model and the last part was to evaluate the structural relationships among causes (IV) and effects (DV).

\subsection{Demographic profile of experts for model verification}

The first part of the question is to verify whether the participants have the attributes of experts by introducing questions on their experience and positions in their companies.

Table 17 shows the demographic profile of experts who participated in the model verification process. It is shown that all the experts have been working in construction for more than 10 years and have been assigned for managerial positions in their corporations. Furthermore, all experts have obtained bachelor's degrees in civil engineering and some others obtained higher degrees. Due to the profound role of the experts in the construction industry and their academic achievements, their inputs, critic and opinions are a significant contribution to this study. Furthermore, the prescribed attributes of the selected experts qualified them to be the targeted respondents for model verification.

\subsection{Verification of measurement model}

This part introduces the analysis of the results for model verification. The method of collecting the data was by the mean of interviews with each individual expert. The questions contained two parts. The first part was about their profile and the second part on

Table 16.

Relationship level among causes and effects from the model

\begin{tabular}{|c|c|c|c|}
\hline $\begin{array}{l}\text { Causes of poor } \\
\text { communication }\end{array}$ & High & $\begin{array}{l}\text { fect factors relationship level } \\
\text { Medium }\end{array}$ & Low \\
\hline $\begin{array}{l}\text { Organizational and } \\
\text { management }\end{array}$ & Performance deficiency & $\begin{array}{l}\text { Unproductive } \\
\text { practitioners }\end{array}$ & Process interruption \\
\hline Project information & $\begin{array}{l}\text { Unproductive } \\
\text { practitioners }\end{array}$ & Performance deficiency & Process interruption \\
\hline $\begin{array}{l}\text { Behavioral and } \\
\text { cultural }\end{array}$ & $\begin{array}{l}\text { Unproductive } \\
\text { practitioners }\end{array}$ & Process interruption & Performance deficiency \\
\hline $\begin{array}{l}\text { Technology and } \\
\text { methods }\end{array}$ & Process interruption & $\begin{array}{l}\text { Unproductive } \\
\text { practitioners }\end{array}$ & Performance deficiency \\
\hline
\end{tabular}

\begin{tabular}{lllr}
\hline Experts & Qualification & Position & Experience (years) \\
\hline E1 & Bachelor's degree & Project Manager & $>15$ \\
E2 & Bachelor's degree & Senior Architect & 13 \\
E3 & Bachelor degree & Senior Engineer & $>11$ \\
E4 & Master degree & Project Engineer & $>16$ \\
E5 & Bachelor degree & Project Manager & 17 \\
E6 & Bachelor degree & Senior Designer & 18 \\
E7 & Master degree & Executive Project Manager & $>25$ \\
E8 & Bachelor degree & Senior Quantity Surveyor & $>20$ \\
E9 & Bachelor degree & Project Structural Engineer & $>15$ \\
E10 & Master & Project Manager & $>20$ \\
E11 & Bachelor degree & Senior Planning Engineer & $>25$ \\
E12 & Bachelor degree & Senior Designer & $>10$ \\
E13 & Bachelor degree & Principal Engineer & $>20$ \\
E14 & Bachelor degree & Project Manager & $>13$ \\
\hline
\end{tabular}

Table 17. $\quad$ E

Experts'

demography for $\quad$ E13

model verification

E14 
the model verification. The model architecture was briefly explained to them prior to asking them questions then the participants were given enough time to answer the second part after that the open-ended questions were raised to them on the model applicability and uses in the construction project lifecycle. The result is divided into two parts in which the first part focused on the verification of the measurement model and the second part discussed the structural model. The experts were asked to agree or disagree or else suggest a new ranking based on their experiences. Table 18 illustrates the verification of the measurement model for cause factors.

Table 18 shows the results of measurement model verification for causes factors, which were attained from the expert's viewpoint. From the results, most of the experts agreed upon the outcomes produced from the PLS-SEM model. Nevertheless, few experts disagreed about the ranking of some factors, which they believe either the rank should be for all the groups, the first ranking factor for each group was also confirmed by the expert and all of them agreed upon the model findings. However, for the second-ranked factor, not all the experts agreed on the outcome. For the first group (OM), only 11 experts agreed that contractual barriers (restrictions) ranked as the second, but the rest 4 experts disagreed on

\begin{tabular}{|c|c|c|c|c|c|c|}
\hline Group & Cause factors & Ranking & & $\begin{array}{l}\text { ree } \\
(\%)\end{array}$ & & $\begin{array}{r}\text { gree } \\
(\%)\end{array}$ \\
\hline \multirow{11}{*}{$\begin{array}{l}\text { Organizational and } \\
\text { management }\end{array}$} & Infirm organizational communication structure & 1 & 14 & 100 & 0 & 0 \\
\hline & Contractual barriers (restrictions) & 2 & 11 & 79 & 3 & 21 \\
\hline & Lack of formal execution of communication & 3 & 12 & 86 & 2 & 14 \\
\hline & Poor project information management & 4 & 12 & 86 & 2 & 14 \\
\hline & Lack of communication plan & 5 & 13 & 93 & 1 & 7 \\
\hline & Lack of communication skills & 6 & 13 & 93 & 1 & 7 \\
\hline & Different level of education & 7 & 11 & 79 & 3 & 21 \\
\hline & Improper project planning & 8 & 10 & 71 & 4 & 29 \\
\hline & Different level of technical skills & 9 & 12 & 86 & 2 & 14 \\
\hline & Frequent change of project contract & 10 & 13 & 93 & 1 & 7 \\
\hline & Lack of collaboration and representation & 11 & 11 & 79 & 3 & 21 \\
\hline \multirow[t]{4}{*}{ Project information } & Inaccessibility of project information & 1 & 14 & 100 & 0 & 0 \\
\hline & Lack of timely information & 2 & 14 & 100 & 0 & 0 \\
\hline & Poor project documentation & 3 & 12 & 86 & 2 & 14 \\
\hline & Unavailability of crucial information & 4 & 13 & 93 & 1 & 7 \\
\hline \multirow{8}{*}{$\begin{array}{l}\text { Behavioral and } \\
\text { cultural }\end{array}$} & Lack of confidence & 1 & 14 & 100 & 0 & 0 \\
\hline & Language barrier & 2 & 12 & 86 & 2 & 14 \\
\hline & Diversity of culture & 3 & 13 & 93 & 1 & 7 \\
\hline & Negligence and casualness & 4 & 13 & 93 & 1 & 7 \\
\hline & Lack of ethics & 5 & 14 & 100 & 0 & 0 \\
\hline & Fear to communicate & 6 & 14 & 100 & 0 & 0 \\
\hline & Lack of understanding and acceptance & 7 & 14 & 100 & 0 & 0 \\
\hline & Usage of different terminologies & 8 & 12 & 86 & 2 & 14 \\
\hline \multirow{6}{*}{$\begin{array}{l}\text { Technology and } \\
\text { methods }\end{array}$} & Improper introduction of new technology Ex. (BIM) & 1 & 14 & 100 & 0 & 0 \\
\hline & Lack of interaction among different discipline & 2 & 13 & 93 & 1 & 7 \\
\hline & Lack of standardization of progress measurement & 3 & 14 & 100 & 0 & 0 \\
\hline & Lack of communication system and platform & 4 & 14 & 100 & 0 & 0 \\
\hline & Improper communication channel & 5 & 13 & 93 & 1 & 7 \\
\hline & Lack of appropriate communication medium & 6 & 12 & 86 & 2 & 14 \\
\hline
\end{tabular}

Notes: Remarks: \#: frequency; \%: percentage
Causes and effects of poor communication 
JFM

21,1

138

this finding. This is justified based on their opinions some other factors are more important to be considered as the second-ranked in the group.

The second group is causes related to PI. From the results, the agreement percentage in the interval of 100 to 86 minimum and this is shown equivalent agreement between expert's verification and model outcomes. The third group is BC-related causes. It is shown the minimum disagreement percentage is 86 which is relatively acceptable and, thus the group expert verification is in line with the model outcomes. The same applied to the fourth group which is TM related causes and the agreement is more than $50 \%$ and that is exhibited similar outcomes with the model.

As an overall result, the disagreement on the findings in most cases is less than $50 \%$ of the experts and that shows the outcome of the model is verified as most experts confirmed the results obtained from the model. The second part is to verify the ranking of effect factors.

Table 19 presents the verification results obtained from experts who participated in the model verification process. The table shows the ranking of the effect factors of poor communication in construction based on their corresponding groups. From the results, most of the experts agreed upon the outcomes produced by the model. Hence, the ranking of effect factors is verified and both results produced from the model and experts are correspondingly equivalent and accepted for further discussions. The following part is to verify the relationship between IV (causes) and DV (effects) based on the path coefficient level produced by the model analysis.

\subsection{Verification of structural model}

This section outlines the assessment of the cause-and-effect relationships based on the outcome produced by the coefficient of the path model. The cause factors are considered the $\mathrm{IV}$, which have a relationship to impact on the effect factors (DVs). The relationship level is

\begin{tabular}{|c|c|c|c|c|c|c|}
\hline Group & Effect factors & Ranking & $\begin{array}{r}\mathrm{Ag} \\
\text { freq }\end{array}$ & $\begin{array}{l}\text { ree } \\
(\%)\end{array}$ & $\begin{array}{l}\text { Disa } \\
\text { freq }\end{array}$ & $\begin{array}{l}\text { gree } \\
(\%)\end{array}$ \\
\hline \multirow{4}{*}{$\begin{array}{l}\text { Unproductive } \\
\text { practitioners }\end{array}$} & Demotivation & 1 & 14 & 100 & 0 & 0 \\
\hline & High stress in the workplace & 2 & 14 & 100 & 0 & 0 \\
\hline & Low level of satisfaction & 3 & 13 & 93 & 1 & 7 \\
\hline & Deteriorating relationship and cooperation & 4 & 14 & 100 & 0 & 0 \\
\hline \multirow{9}{*}{$\begin{array}{l}\text { Performance } \\
\text { deficiency }\end{array}$} & Waste generation & 1 & 13 & 93 & 1 & 7 \\
\hline & Safety standard violations & 2 & 14 & 100 & 0 & 0 \\
\hline & Low productivity & 3 & 14 & 100 & 0 & 0 \\
\hline & Poor collaboration & 4 & 13 & 93 & 1 & 7 \\
\hline & Cost overrun & 5 & 14 & 100 & 0 & 0 \\
\hline & Rework occurrence & 6 & 13 & 93 & 1 & 7 \\
\hline & Failure of the project & 7 & 14 & 100 & 0 & 0 \\
\hline & Dispute & 8 & 12 & 86 & 2 & 14 \\
\hline & Time overrun & 9 & 13 & 93 & 1 & 7 \\
\hline \multirow[t]{4}{*}{ Process interruption } & Redesign & 1 & 14 & 100 & 0 & 0 \\
\hline & Misinterpreted technical information & 2 & 12 & 86 & 2 & 14 \\
\hline & Frequent remedies in design and planning schedules & 3 & 13 & 93 & 1 & 7 \\
\hline & Information overlapping & 4 & 13 & 93 & 1 & 7 \\
\hline
\end{tabular}

Table 19.

Results of model verification (effect factors)

Notes: freq: frequency; \%: percentage 
justified numerically using the coefficient of path models produced from the model analysis and the confirmation of the predeveloped hypothetical model in the previous sections.

Table 20 illustrates the verification results of cause-and-effect relationships. From the results, all the experts agreed that the causes factors under OM have strong performance deficiency and 13 experts agreed that it has a medium relationship with PRA-related effects and 12 agreed that it has a small effect with PRO. As an overall conclusion, most experts agreed upon the results produced by the model and the relationships are verified accordingly.

\subsection{Model application}

This section explores an opportunity on applying the model to construction activities among the same selected experts as in Section 17. This exploration was conducted by showing the model to the experts and requesting the expert to share their opinion on the possibility of applying the model to the construction activity. According to the experts, the model is likely to be applied in different three stages of the project including planning, execution and project closure.

Table 21 shows the outcomes of the model applications in the stages of planning, execution and closure. All the experts agreed the model can be applied in the planning stage and the possible applications can be during developing a communication plan whereby the model is likely to be used to induce more attention to dominant causative factors to produce an effective communication plan. Experts also added the model is a beneficial guideline to introduce comprehensive understanding to main parties to improve and smooth communication exchange. It was also revealed; the outcome of the model is also useful to individual technical project participants including managers and engineers whereby they can be able to identify the issues that hindered the production of effective communication. However, during the execution stage where many activities are executed concurrently and that makes the communication and information exchange more complex and intricate and a clash is likely to occur and rework may happen. According to the experts, the model is possibly applied in the construction stage in the following context:

- The outcome prioritizes the causes of poor communication by highlighting the most critical causes and help them to take precautionary actions to avoid the causes.

- The model also uncovered the effects of poor communication and, thus help construction practitioners to consider what if the communication is not properly executed, thus avoiding the causes.

- The model established the classification of the causes and effects to different groups, hence it helps the decision-makers to relate the factors to their respective categories.

- The model is likely to be applied to assess the health status of communication during the project execution.

Regarding the closure stage, which is the final stage of the construction project and it is a critical stage where the contractor is expediting to finish the project on time and huge PI is to be archived accordingly. According to the expert, the model can be applied in the following aspects:

- The model helps construction parties to understand what causes the communication interruption, thus defining pre-measures to produce a more efficient information exchange process.

- Assessment of communication status based on the causes and effects significances.
Causes and effects

\section{of poor \\ communication}

. 
JFM

21,1

140

Table 20.

Matrix of cause-and-

effect group

relationships

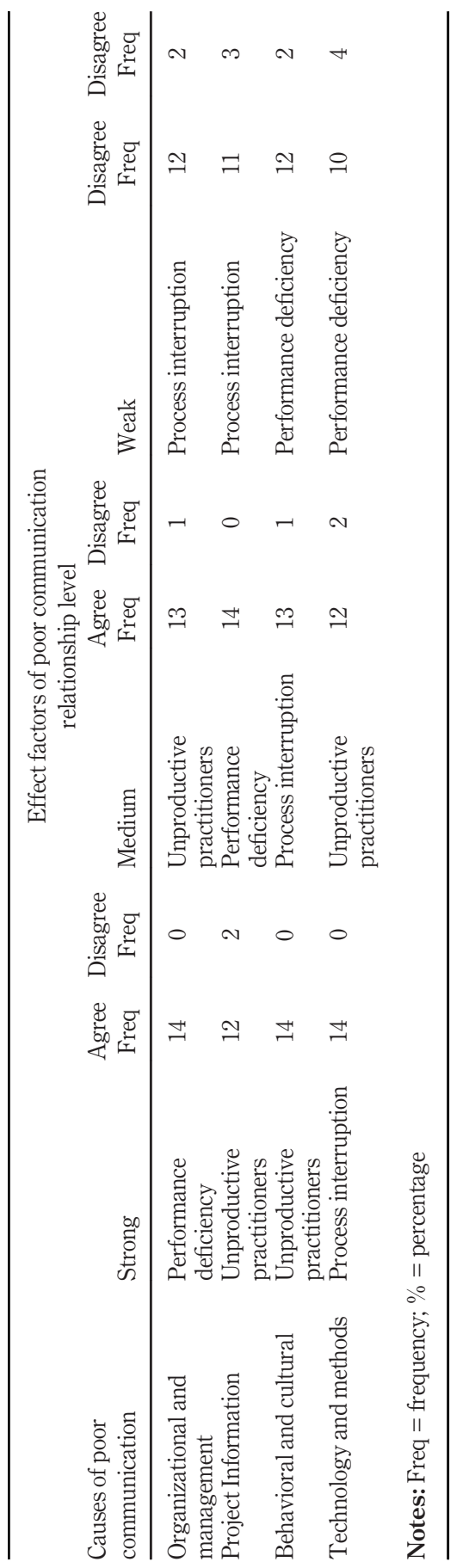




\begin{tabular}{|c|c|c|c|c|}
\hline Stage Exp & Planning & Execution & Closure & of poor \\
\hline $\begin{array}{l}\text { E1 } \\
\text { E2 }\end{array}$ & $\sqrt{ }$ & $\sqrt{ }$ & & communication \\
\hline $\begin{array}{l}\text { E2 } \\
\text { E3 }\end{array}$ & $\sqrt{ }$ & & $\sqrt{ }$ & \\
\hline $\begin{array}{l}\text { E3 } \\
\text { E4 }\end{array}$ & $\sqrt{ }$ & & $\sqrt{ }$ & \\
\hline E5 & $\sqrt{V}$ & $\sqrt{ }$ & & \\
\hline E6 & $\sqrt{ }$ & $\mathrm{v}$ & $\sqrt{ }$ & 141 \\
\hline $\begin{array}{l}\text { E7 } \\
\text { E8 }\end{array}$ & $\sqrt{ }$ & & $\sqrt{ }$ & \\
\hline $\begin{array}{l}\text { E8 } \\
\text { E9 }\end{array}$ & $\sqrt{ }$ & & $\sqrt{ }$ & \\
\hline E10 & $\sqrt{ }$ & $\sqrt{ }$ & $\sqrt{v}$ & \\
\hline E11 & $\sqrt{ }$ & $\sqrt{ }$ & $\sqrt{ }$ & \\
\hline E12 & $\sqrt{ }$ & $\sqrt{ }$ & $\sqrt{ }$ & Table 21 . \\
\hline E13 & $\sqrt{ }$ & $\sqrt{ }$ & & Applications of the \\
\hline E14 & $\sqrt{14}$ & $\sqrt{ }$ & & model-based on \\
\hline Frequency & 14 & 8 & 9 & experts' opinions \\
\hline
\end{tabular}

\section{Conclusion}

This article introduced the development of the PLS-SEM model of cause-and-effect factors of poor communication in the construction industry using Smart-PLS 3 which is used to study the relationship among causes and effects, which has been identified from previously published literature (Gamil and Rahman, 2017). The hypothetical model consists of 12 hypotheses and 7 constructs which 4 of them are considered as independent cause factors and 3 as dependent effect factors. The model developed has undergone a thorough assessment process for both model's parts, which are measurement and structural model. At the initial stage iteration process was adopted to delete indicators, which have less item reliability and loadings factors, After the second iteration, the measurement model achieved the required criterion for convergent validity than the model used for discriminant validity using cross-loading and Fornell-Larcker criterion. After finalizing the assessment of the measurement model, the structural model is assessed for all path modeling and hypothesis. The model was also assessed for global GoF and the results achieved the requirement prescribed by Cohen (2013) and Wetzels et al. (2009) and the model resulted in $0.38 \mathrm{GoF}$, which is considered as large enough to be considered to obtain sufficient global PLS model validity. From the final model presented in Figure 9, it can be concluded that there is a possible relationship between IV and DV, which is illustrated by the path model verified. The model has been also verified by 14 construction experts. An explanation of the model was introduced prior to answering the question then experts were asked to answer the predefined questions and give their thoughts on the model. The results show that most experts agreed on the model outcomes. Therefore, the results have been verified practically and the outcome of this part assumes to help the construction players and other construction industry policymakers to understand the causes and effects of poor communication and their associated relationship to achieve maximum comprehension of the phenomenon of poor communication in the industry. To conclude, the developed model is significant for construction parties to understand the relationship between cause-and-effect factors of poor communication in the construction industry. It is also significant for construction practitioners in all perspectives to understand the phenomenon of poor communication in the construction industry. 


\section{References}

Ab Hamid, M., Sami, W. and Sidek, M.M. (2017), "Discriminant validity assessment: use of Fornell and Larcker criterion versus HTMT criterion", Paper presented at the Journal of Physics: Conference Series.

Abd El-Razek, M., Bassioni, H. and Mobarak, A. (2008), "Causes of delay in building construction projects in Egypt", Joumal of Construction Engineering and Management, Vol. 134 No. 11, pp. 831-841.

Abdullah, M.R., Rahman, I.A. and Azis, A.A.A. (2009), "Delay in large mara construction projects based on project management consultant perspective", Paper presented at the Proc. Malaysian Technical Universities Conference on Engineering and Technology.

Abdul-Rahman, H. (1995), "The cost of non-conformance during a highway project: a case study", Construction Management and Economics, Vol. 13 No. 1, pp. 23-32.

Abusafiya, H.A. and Suliman, S. (2017), "Causes and effects of cost overrun on construction project in Bahrain: part 2 (PLS-SEM path modelling)”, Modern Applied Science, Vol. 11 No. 7, pp. 28-37.

Adnan, H. (2004), Joint Venture Projects in Malaysian Construction Industry: factors Critical to Success, Nottingham Trent University (United Kingdom).

Adnan, H., Shamsuddin, S.M., Supardi, A. and Ahmad, N. (2012), "Conflict prevention in partnering projects”, Procedia - Social and Behavioral Sciences, Vol. 35, pp. 772-781.

Affare, M.A.W. (2012), “An assessment of project communication management on construction projects in Ghana".

Afshari, H., Khosravi, S., Ghorbanali, A., Borzabadi, M. and Valipour, M. (2010), "Identification of causes of non-excusable delays of construction projects", Paper presented at the International conference on e-business management and economics.

Ahuja, V. (2007), IT Enhanced Communication Protocols for Building Project Management by Small and Medium Enterprises in the Indian Construction Industry, Queensland University of Technology.

Aiyewalehinmi, E. (2013), "Factor analysis of communication in the construction industry", The International Journal of Engineering and Science, Vol. 2 No. 10, pp. 49-57.

Alaghbari, W.e., Kadir, M.R.A. and Salim, A. (2007), "The significant factors causing delay of building construction projects in Malaysia", Engineering, Construction and Architectural Management.

Alhomidan, A. (2013), "Factors affecting cost overrun in road construction projects in Saudi Arabia", International Journal of Civil and Environmental Engineering, Vol. 13 No. 3, pp. 1-4.

Ali, A.S. and Wen, K.H. (2011), "Building defects: possible solution for poor construction workmanship", Journal of Building Performance, Vol. 2 No. 1.

Al-Najjar, J.M. (2008), "Factors influencing time and cost overruns on construction projects in the Gaza Strip".

Alwi, S., Mohamed, S. and Hampson, K. (2002), "Waste in the Indonesian construction projects", Paper presented at the Proceedings of the 1st CIB-W107 International Conference-Creating a Sustainable Construction Industry in Developing Countries.

Apolot, R.E. (2011), Investigation of the Causes of Delay and Cost Overrun in Construction Projects in Uganda's Public Sector, Makerere University.

Armstrong, M. and Taylor, S. (2020), “Armstrong's handbook of human resource management practice".

Arnorsson, H. (2012), "Optimizing the information flow on the construction site", Masterąŕs Thesis, Faculty of Engineering and Science, Aalborg University, Denmark.

Astrachan, C.B., Patel, V.K. and Wanzenried, G. (2014), "A comparative study of CB-SEM and PLSSEM for theory development in family firm research", Journal of Family Business Strategy, Vol. 5 No. 1, pp. 116-128.

Aulich, T. (2013), "The role of effective communication in the construction industry: a guide for education and health clients", Construction Economics and Building, Vol. 13 No. 4, pp. 92-101. 
Aziz, R.F. and Hafez, S.M. (2013), "Applying lean thinking in construction and performance improvement", Alexandria Engineering Journal, Vol. 52 No. 4, pp. 679-695.

Badu, E., Fugar, F. and Assah-Kissiedu, M. (2010), "Triggers of disputes within the ghanaian construction industry".

Causes and effects of poor communication

Baguley, P. (1994), Effective Communication for Modern Business, McGraw-Hill.

Ballan, B. (2011), "A value map for communication systems in construction”, Journal of Information Technology in Construction (ITcon), Vol. 16 No. 44, pp. 745-760.

Bandulahewa, B.K.M. (2015), "Effective project communication for construction project managers in Sri Lanka”.

Bassioni, H.A., Sarhan, A. and Zaki, A.S. (2013), "Cost overrun causes related to the design phase in the Egyptian construction industry", Journal of Engineering Management Research, Vol. 1 No. 5, pp. 138-147.

Batool, A. and Abbas, F. (2017), "Reasons for delay in selected hydro-power projects in Khyber Pakhtunkhwa (KPK), Pakistan”, Renewable and Sustainable Energy Reviews, Vol. 73, pp. 196-204.

Best, R. and De Valence, G. (2007), Design and Construction, Routledge.

Bowen, N.K. and Guo, S. (2011), Structural Equation Modeling, Oxford University Press.

BSI (2003), Poor Communication Costing UK Construction Industry, Press Release, British Standards Institution.

Buchanan, D.A. and Huczynski, A.A. (2019), Organizational Behavior, Pearson.

Buys, F. and Le Roux, M. (2013), "Causes of defects in the South African housing construction industry: perceptions of built-environment stakeholders: review articles", Acta Structilia: Journal for the Physical and Development Sciences, Vol. 20 No. 2, pp. 78-99.

Cakmak, P.I. and Cakmak, E. (2013), "An analysis of causes of disputes in the construction industry using analytical hierarchy process (AHP)", AEI 2013: Building Solutions for Architectural Engineering, pp. 94-102.

Chan, D.W. and Kumaraswamy, M.M. (1997), "A comparative study of causes of time overruns in Hong Kong construction projects", International Journal of Project Management, Vol. 15 No. 1, pp. 55-63.

Chen, J.J. (1998), "The characteristics and current status of China's construction industry", Construction Management and Economics, Vol. 16 No. 6, pp. 711-719.

Cheng, E.W., Li, H., Love, P.E. and Irani, Z. (2001), "Network communication in the construction industry", Corporate Communications: An International Journal, Vol. 6 No. 2.

Chin, W.W. (1998), "The partial least squares approach to structural equation modeling", Modern Methods for Business Research, Vol. 295 No. 2, pp. 295-336.

Chong, H.Y. and Low, T.S. (2014), "Accidents in malaysian construction industry: statistical data and court cases", International Journal of Occupational Safety and Ergonomics, Vol. 20 No. 3, pp. 503-513.

Cohen, J. (2013), Statistical Power Analysis for the Behavioral Sciences, Academic press.

Coopers (2012), "Insights and trends: Current portfolio, programme and project management practices. Price Waterhouse Coopers", A report, available at: www.pmi.org/ /media/PDF/RCP/ PwC_PPPM_Trends_2012.ashx

Coughlan, J.M.M. (2008), "Structural equation modelling: guidelines for determining model fit", Electronic Journal of Business Research Methods, Vol. 6 No. 1, pp. 53-60.

Crowe, A. (2005), The PMP Exam: How to Pass on Your First Try, Velociteach Press.

Dainty, A., Moore, D. and Murray, M. (2007), Communication in Construction: Theory and Practice, Routledge. 
Darvik, L. and Larsson, J. (2010), “The impact of material delivery-deviations on costs and performance in construction projects".

Dingsdag, D., Biggs, H. and Sheahan, V. (2006), "Safety culture in the construction industry: changing behaviour through enforcement and education", Paper presented at the Clients Driving Innovation: Moving Ideas into Practice: Proceedings of the CRCCI 2nd International Conference.

Dubois, A. and Gadde, L.E. (2002), "The construction industry as a loosely coupled system: implications for productivity and innovation”, Construction Management and Economics, Vol. 20 No. 7, pp. 621-631.

Emmitt, S. and Gorse, C. (2006), Communication in Construction Teams, Routledge.

Emuze, F. and James, M. (2013), "Exploring communication challenges due to language and cultural diversity on South African construction sites", Acta Structilia: Journal for the Physical and Development Sciences, Vol. 20 No. 1, pp. 44-65.

Enshassi, A. and Al Swaity, E. (2015), "Key stressors leading to construction professionals' stress in the Gaza Strip", Palestine. Journal of Construction in Developing Countries, Vol. 20 No. 2.

Enshassi, A., Al-Hallaq, K. and Mohamed, S. (2006), "Causes of contractor's business failure in developing countries: the case of Palestine", Journal of Construction in Developing Countries, Vol. 11 No. 2.

Falk, R.F. and Miller, N.B. (1992), A Primer for Soft Modeling, University of Akron Press.

Faridi, A.S. and El-Sayegh, S.M. (2006), "Significant factors causing delay in the UAE construction industry", Construction Management and Economics, Vol. 24 No. 11, pp. 1167-1176.

Fayek, A.R., Dissanayake, M. and Campero, O. (2003), "Measuring and classifying construction field rework: a pilot study", Research Rep, (May).

Fichet, H. and Giraud, L. (2007), How the Information Flow is Processed in Project-Based Companies Compared to Others and How It Affects Strategic Drift, Handelshögskolan vid Umeå universitet.

Fornell, C. (1994), "Partial least squares. Advanced methods of marketing research".

Fornell, C. and Larcker, D.F. (1981), Structural Equation Models with Unobservable Variables and Measurement Error: Algebra and Statistics, Sage Publications, Los Angeles, CA.

Gamil, Y., Abd Rahman, I. and Nagapan, S. (2019), "Investigating the effect of poor communication in terms of cost and time overruns in the construction industry", Management, Vol. 9 No. 2, pp. 94-106.

Gamil, Y. and Rahman, I.A. (2017), "Identification of causes and effects of poor communication in construction industry: a theoretical review", Emerging Science Journal, Vol. 1 No. 4, pp. 239-247.

Garson, G.D. (2012), Testing Statistical Assumptions, Statistical Associates Publishing, Asheboro, Nc.

Gebken, R.J. and Gibson, G.E. (2006), "Quantification of costs for dispute resolution procedures in the construction industry", Journal of Professional Issues in Engineering Education and Practice, Vol. 132 No. 3, pp. 264-271.

Guide, A. (2001), "Project management body of knowledge (pmbok® guide)", Paper presented at the Project Management Institute.

Günhan, S., Şenol, G. and Doğan, S.Z. (2012), "Non-verbal cues: improving communication in construction projects", Paper presented at the 2012 ASEE annual conference program: final conference program and proceedings.

Hair, J.F., Jr, Hult, G.T.M., Ringle, C.M. and Sarstedt, M. (2021), A Primer on Partial Least Squares Structural Equation Modeling (PLS-SEM), Sage publications.

Hammarlund, Y. and Josephson, P. (1991), "Sources of quality failures in building”, Paper presented at the Proc., European Symp. on Management, Quality and Economics in Housing and other Building Sectors.

Haslam, R.A., Hide, S.A., Gibb, A.G., Gyi, D.E., Pavitt, T., Atkinson, S. and Duff, A.R. (2005), "Contributing factors in construction accidents", Applied Ergonomics, Vol. 36 No. 4, pp. 401-415. 
Henderson, L.S., Stackman, R.W. and Lindekilde, R. (2016), "The centrality of communication norm alignment, role clarity, and trust in global project teams", International Journal of Project Management, Vol. 34 No. 8, pp. 1717-1730.

Henseler, J., Hubona, G. and Ray, P.A. (2016), "Using PLS path modeling in new technology research: updated guidelines", Industrial Management and Data Systems, Vol. 116 No. 1.

Henseler, J., Ringle, C.M. and Sarstedt, M. (2015), "A new criterion for assessing discriminant validity in variance-based structural equation modeling", Journal of the Academy of Marketing Science, Vol. 43 No. 1, pp. 115-135.

Hoezen, M., Reymen, I. and Dewulf, G. (2006), “The problem of communication in construction”, Paper presented at the CIB W96 Adaptable Conference, University of Twente.

Hossain, L. (2009), "Communications and coordination in construction projects", Construction Management and Economics, Vol. 27 No. 1, pp. 25-39.

Iacobucci, D. (2010), "Structural equations modeling: fit indices, sample size, and advanced topics", Journal of Consumer Psychology, Vol. 20 No. 1, pp. 90-98.

Isa, R.B., Jimoh, R.A. and Achuenu, E. (2013), "An overview of the contribution of construction sector to sustainable development in Nigeria”, Net Journal of Business Management, Vol. 1 No. 1, pp. 1-6.

Jureddi, D.N. and Brahmaiah, N. (2016), "Barriers to effective communication", Journal of English Language and Literature, Vol. 3 No. 2, pp. 114-115.

Kassab, M., Hipel, K. and Hegazy, T. (2006), "Conflict resolution in construction disputes using the graph model", Journal of Construction Engineering and Management, Vol. 132 No. 10, pp. 1043-1052.

Kent, D.C. and Becerik-Gerber, B. (2010), "Understanding construction industry experience and attitudes toward integrated project delivery", Journal of Construction Engineering and Management, Vol. 136 No. 8, pp. 815-825.

Kent, K. (1996), "Communication as a core management discipline: the relationship between new management trends and the need for new perspectives in education in both management and public relations", Journal of Communication Management, Vol. 1 No. 1.

Khahro, S.H. and Ali, T.H. (2014), "Causes leading to conflicts in construction projects: a viewpoint of Pakistani construction industry", Paper presented at the International Conference on challenges in IT, Engineering and Technology (ICCIET'2014) July.

Kock, N. and Hadaya, P. (2018), "Minimum sample size estimation in PLS-SEM: the inverse square root and gamma-exponential methods", Information Systems Journal, Vol. 28 No. 1, pp. 227-261.

Kornelius, L. and Wamelink, J. (1998), “The virtual corporation: learning from construction”, Supply Chain Management: An International Journal, Vol. 3 No. 4.

Kuang, C.H., Wong, N.L. and David, M.K. (2010), “Communication mode among Malaysians”.

Lee, J. and Bernold, L.E. (2008), "Ubiquitous agent-based communication in construction”, Journal of Computing in Civil Engineering, Vol. 22 No. 1, pp. 31-39.

Liao, P.C., Lei, G., Fang, D. and Liu, W. (2014), "The relationship between communication and construction safety climate in China", KSCE Journal of Civil Engineering, Vol. 18 No. 4, pp. 887-897.

Lohiya, G. (2012), "Team building in project management practice in the UAE construction industry", International Proceedings of Economics Development and Research, Vol. 43, pp. 31-35.

Loosemore, M. and Lee, P. (2002), "Communication problems with ethnic minorities in the construction industry", International Journal of Project Management, Vol. 20 No. 7, pp. 517-524.

Love, P.E. and Li, H. (2000), "Quantifying the causes and costs of rework in construction", Construction Management and Economics, Vol. 18 No. 4, pp. 479-490.

Malleson, A. (2013), "National construction contracts and law survey 2013”, pp. 08-21.

Causes and effects of poor communication

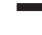


Memon, A.H. and Rahman, I.A. (2014), "SEM-PLS analysis of inhibiting factors of cost performance for large construction projects in Malaysia: perspective of clients and consultants", The Scientific World Journal, Vol. 2014.

Mohamad, M.M., Sulaiman, N.L., Sern, L.C. and Salleh, K.M. (2015), "Measuring the validity and reliability of research instruments", Procedia - Social and Behavioral Sciences, Vol. 204, pp. 164-171.

Nagapan, S. (2014), Structural Modelling of Cause and Effect Factors of Conrtsuction Waste Generation in Malaysian Construction Industry, Universiti Tun Hussein Onn Malaysia.

Nguyen, T.P. and Chileshe, N. (2013), Revisiting the Critical Factors Causing Failure of Construction Projects in Vietnam, Association of Researchers in Construction Management.

Nylén, K.O. (1996), Cost of Failure in a Major Civil Engineering Project, KTH, Stockholm.

Ofori, G. and Ekanayake, L. (2000), "Construction material waste source evaluation”, Paper presented at the Proceedings of the Second Southern African Conference on Sustainable Development in the Built Environment, Pretoria.

Olanrewaju, A., Tan, S.Y. and Kwan, L.F. (2017), "Roles of communication on performance of the construction sector", Procedia Engineering, Vol. 196, pp. 763-770.

Olawale, Y.A. and Sun, M. (2010), "Cost and time control of construction projects: inhibiting factors and mitigating measures in practice", Construction Management and Economics, Vol. 28 No. 5, pp. 509-526.

Oluwaseun Sunday, D. and Olumide Afolarin, A. (2013), "Causes, effects and remedies of errors in Nigerian construction documents", Organization, Technology and Management in Construction: An International Journal, Vol. 5 No. 1, pp. 676-686.

Oshodi Olalekan, S. and Rimaka, I. (2013), "A comparative study on causes and effects of delay in Nigerian and Iranian construction projects", Asian Journal of Business and Management Sciences, Vol. 3 No. 1, pp. 29-36.

Othman, N. and Mydin, M. (2014), "Poor workmanship in construction of low cost housing", Analele Universitatii' Eftimie Murgu', Vol. 21 No. 1.

Pasley, G.P. and Kim Roddis, W. (1994), "Using artificial intelligence for concurrent design in the steel building industry", Concurrent Engineering, Vol. 2 No. 4, pp. 303-310.

Pérez Gómez-Ferrer, A. (2017), “Communication problems between actors in construction projects”.

Peterson, R.A. and Kim, Y. (2013), "On the relationship between coefficient alpha and composite reliability”, Journal of Applied Psychology, Vol. 98 No. 1, p. 194.

PMI (2013), "More than half of all project budget risk is due to ineffective communications", available at: www.pmi.org

Pozin, M.A.A., Nawi, M.N.M., Lee, A. and Azman, M. (2018), "Improving communication in managing industrialised building system (IBS) projects: virtual environment", Malaysian Construction Research Journal, Vol. 2 No. 2, pp. 1-13.

Quill, T.E. (1995), Barriers to Effective Communication the Medical Interview, Springer, pp. 110-121.

Rahman, I.A., Memon, A.H., Karim, A.T.A. and Tarmizi, A. (2013), "Significant factors causing cost overruns in large construction projects in Malaysia”, Journal of Applied Sciences, Vol. 13 No. 2, pp. 286-293.

Ren, Z., Atout, M. and Jones, J. (2008), "Root causes of construction project delays in Dubai", Paper presented at the Procs 24th Annual ARCOM Conference, September.

Rigdon, E.E., Ringle, C.M. and Sarstedt, M. (2010), "Structural modeling of heterogeneous data with partial least squares", Review of marketing research.

Ross, C.S. and Dewdney, P. (1998), "Negative closure: strategies and counter-strategies in the reference transaction", Reference and User Services Quarterly, pp. 151-163. 
Rostami, A. and Oduoza, C.F. (2017), 'Key risks in construction projects in Italy: contractors' perspective", Engineering, Construction and Architectural Management, Vol. 24 No. 3.

Salleh, N.A.B., Nordin, N.B.M. and Rashid, A.K.B.A. (2012), "The language problem issue among foreign workers in the Malaysian construction industry", International Journal of Business and Social Science, Vol. 3 No. 11.

Sambasivan, M. and Soon, Y.W. (2007), "Causes and effects of delays in Malaysian construction industry", International Journal of Project Management, Vol. 25 No. 5, pp. 517-526.

Sandelowski, M. (2000), "Combining qualitative and quantitative sampling, data collection, and analysis techniques in mixed-method studies", Research in Nursing and Health, Vol. 23 No. 3, pp. 246-255.

Senaratne, S. and Ruwanpura, M. (2016), "Communication in construction: a management perspective through case studies in Sri Lanka", Architectural Engineering and Design Management, Vol. 12 No. 1, pp. 3-18.

Sharma, G. (2017), "Pros and cons of different sampling techniques", International Journal of Applied Research, Vol. 3 No. 7, pp. 749-752.

Shehu, Z., Endut, I.R., Akintoye, A. and Holt, G.D. (2014), "Cost overrun in the Malaysian construction industry projects: a deeper insight”, International Journal of Project Management, Vol. 32 No. 8, pp. $1471-1480$.

Sinesilassie, E., Tabish, S. and Jha, K. (2018), "Critical factors affecting cost performance: a case of Ethiopian public construction projects", International Journal of Construction Management, Vol. 18 No. 2, pp. 108-119.

Sinha, M. and Wayal, A. (2007), "Dispute causation in construction projects", IOSR Journal of Mechanical and Civil Engineering (IOSR-JMCE).

Tai, S., Wang, Y. and Anumba, C. (2009), "A survey on communications in large-scale construction projects in China”, Engineering, Construction and Architectural Management, Vol. 16 No. 2.

Taleb, H., Ismail, S., Wahab, M. and Rani, M. (2017), "Managing communication between architects and clients during design phase", Malaysian Construction Research Journal, Vol. 2 No. 2, pp. 69-79.

Tenenhaus, M., Vinzi, V.E., Chatelin, Y.M. and Lauro, C. (2005), "PLS path modeling", Computational Statistics and Data Analysis, Vol. 48 No. 1, pp. 159-205.

Thomas, S.R., Tucker, R.L. and Kelly, W.R. (1998), "Critical communications variables", Journal of Construction Engineering and Management, Vol. 124 No. 1, pp. 58-66.

Thorpe, T. and Mead, S. (2001), "Project-specific web sites: friend or foe? ", Journal of Construction Engineering and Management, Vol. 127 No. 5, pp. 406-413.

Thunberg, M., Rudberg, M. and Gustavsson, T.K. (2017), "Categorising on-site problems: a supply chain management perspective on construction projects", Construction Innovation, Vol. 17 No. 1.

Tipili, L.G. and Ojeba, P.O. (2014), "Evaluating the effects of communication in construction project delivery in Nigeria", Paper presented at the Proceedings of the Multi-Disciplinary Academic Conference on Sustainable Development.

Torrington, D. and Hall, S. (1998), Human Resource Management and the Personnel Function, Routledge, London.

Turley, F. (2010), “An introduction to PRINCE2®”, Management Plaza.

Valitherm, A. and Rahman, A. (2014), "Communication barrier in Malaysia construction sites", International Journal of Education and Research, Vol. 2 No. 1, pp. 1-10.

Vinzi, V.E., Trinchera, L. and Amato, S. (2010), PLS Path Modeling: From Foundations to Recent Developments and Open Issues for Model Assessment and Improvement Handbook of Partial Least Squares, Springer, pp. 47-82.

Waziri, F. and Khalfan, S. (2014), "Cross-cultural communication in construction industry: how do Chinese firms cross the barriers in Tanzania?".
Causes and effects of poor communication

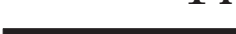


JFM

21,1

Wetzels, M., Odekerken-Schröder, G. and Van Oppen, C. (2009), "Using PLS path modeling for assessing hierarchical construct models: guidelines and empirical illustration", MIS Quarterly, pp. 177-195.

Wong, K.K.K. (2013), "Partial least squares structural equation modeling (PLS-SEM) techniques using SmartPLS”, Marketing Bulletin, Vol. 24 No. 1, pp. 1-32.

Yuan, H. (2012), "A model for evaluating the social performance of construction waste management", Waste Management, Vol. 32 No. 6, pp. 1218-1228.

Zhang, J. (2010), A Social Semantic Web System for Coordinating Communication in the Architecture, Engineering and Construction Industry, University of Toronto.

Zulch, B.G. (2012), The Construction Project Manager as Communicator in the Property Development and Construction Industries, University of the Free State.

Zulch, B.G. (2014), "Communication: the foundation of project management", Procedia Technology, Vol. 16, pp. 1000-1009.

\section{Corresponding author}

Yaser Gamil can be contacted at: Yaser.gamil@1tu.se

For instructions on how to order reprints of this article, please visit our website: 